\title{
Chapter 22 \\ Modeling of the Thermomechanical Behavior, Damage, and Fracture of High Alloy TRIP-Steel
}

\author{
Andreas Seupel, Andreas Burgold, Stefan Prüger, Michael Budnitzki \\ and Meinhard Kuna
}

\begin{abstract}
The aim of this chapter is to give insight into the continuum mechanics based modeling of high alloy TRIP-steels. A powerful thermomechanical framework is presented, which incorporates finite viscoplasticity, the TRIP-effect, complete thermomechanical coupling, and non-local damage. Based on this, different variants of material models are developed. Thereby, selected topics concerning the material behavior of TRIP-steels are examined: Firstly, the mechanical behavior at different temperatures and strain rates is modeled including tension-compression-asymmetry and curve crossing effects. Secondly, the influence of phase transformation on fracture is investigated. Because of the TRIP-effect, higher stresses occur during crack tip blunting. Furthermore, a transformation induced shielding effect is revealed by the evaluation of material forces. Thirdly, damage evolution and crack extension are simulated with a cohesive zone model and with the non-local damage model, respectively. The damage related parameters of these models are determined using available experimental data. The developed numerical models enable quantitative assessments of failure in components made of TRIP-steels.
\end{abstract}

\subsection{Introduction}

In order to optimize design and functionality of components and structures made of TRIP-steels, appropriate macroscopic models for simulation purposes are required. Furthermore, micromechanically informed models can be used in numerical studies to improve the material design itself, e.g., the constitution of composite materials like the particle-reinforced TRIP-matrix composites considered in CRC 799 [1-5].

The particular challenges of modeling the deformation behavior of high alloy TRIP-steels are manifold: Firstly, a considerable variation of mechanical response with changing temperature is observed due to the high influence of temperature on the underlying deformation mechanisms (TRIP-effect: transformation induced plas-

A. Seupel $(\varangle) \cdot$ A. Burgold $\cdot$ S. Prüger $\cdot$ M. Budnitzki $\cdot$ M. Kuna Institute of Mechanics and Fluid Dynamics, Lampadiusstraße 4, 09596 Freiberg, Germany e-mail: Andreas.Seupel@imfd.tu-freiberg.de 
ticity, TWIP-effect: twinning induced plasticity, dislocation glide, [6-8]). A thermomechanical coupling has to be taken into account in order to explain and model loading rate effects basically caused by the temperature dependency, e.g., the curve crossing effect [9, 10]. Another modeling aspect is given by the observed loading state dependent strain hardening of TWIP and TRIP-steels often recognized as tension-compression asymmetry or strength differential effect [11-14]. Moreover, the question arises, how the TRIP-effect influences the damage and fracture behavior of austenitic steels. This becomes of interest in safety applications, metal forming, and lightweight design. Taking damage and material degradation into account, conceptional problems arise: So-called local damage models imply an ill-posed boundary value problem in the classical continuum mechanics framework. A regularization method is necessary to circumvent pathological effects during numerical treatment, e.g., the mesh dependency well known from numerical analyses utilizing the FiniteElement-Method (FEM).

In this paper, two types of material models for high alloy TRIP-steels are presented - each developed to meet different requirements according to the CRC's state-of-theart: Firstly, a micromechanically motivated model is considered and enhanced which is based on an advanced homogenization scheme [15]. Thereby, information on the state and micromechanical features of both phases, austenite and martensite, can be included. In addition, the model exhibits a higher predictive character than purely phenomenological approaches. For example, the model is successfully applied in micromechanical simulations conducted to assess and to improve the properties of particle reinforced TRIP-matrix-composites [16-18]. Furthermore, the model is used in fundamental investigations on fracture mechanics aspects of high alloy TRIP-steel, which reveal the role of martensitic phase transformation on ductility and fracture resistance [19-21]. Moreover, essential enhancements of the micromechanical model are proposed elsewhere, especially to incorporate thermomechanical coupling and stress state dependent hardening behavior of high alloy TRIP-steels [9, 22].

However, due to the high complexity and sophisticated numerical treatment of the micromechanical model, a robust phenomenological alternative is developed as basis for advanced damage modeling. The main aim is to set up a regularized damage model, which does not suffer from pathological localization effects. Simultaneously, a thermomechanically coupled variant of the model is proposed, which includes asymmetric strain hardening.

The paper is organized as follows: In the next section, a thermomechanicalmicromorphic framework for finite deformations is briefly introduced, which is especially used as basis for the regularized damage model and to discuss thermodynamical aspects.

In the third section, the distinct material models are presented. As common starting point, a multiplicative viscoplasticity and martensite kinetics models for the strain induced regime are used.

In the fourth section, modeling results on thermomechanical material response, damage, and fracture are presented. The thermomechanically enhanced phenomenological model is calibrated to experimental results of a cast $\mathrm{X} 3 \mathrm{CrMnNi} 16-6-6$ TRIP-steel developed within the CRC 799. Afterwards, the model is assessed by 
comparing the model predictions of thermomechanical loading and inhomogeneous deformation scenarios to available experiments. Using the micromechanical material model, numerical investigations on influence of the TRIP-effect on crack tip fields and fracture mechanics properties are discussed. Fracture of high alloy TRIP-steels is modeled by a cohesive zone approach, and finally by the micromorphic model of ductile damage.

\subsection{Thermomechanical Framework}

The thermomechanically coupled modeling as well as the regularized damage formulation are set up within the micromorphic framework proposed by Forest [23]. The thermomechanical DOF are the displacement vector $\vec{u}$ and the absolute temperature $\vartheta$. The degrees of freedom (DOF) are enriched by a scalar valued micromorphic variable $\varepsilon_{\mathrm{nl}}$ :

$$
\mathrm{DOF}=\left\{\vec{u}, \vartheta, \varepsilon_{\mathrm{nl}}\right\}
$$

The micromorphic variable has a local counterpart, which should be enhanced by gradient effects.

The local material behavior depends on the evolution of state variables (SV). Inelastic, loading history dependent processes are treated by the thermodynamics of internal variables principle. The chosen state variables read

$$
\mathrm{SV}=\left\{\varepsilon_{\log }^{\mathrm{el}}, \vartheta, \kappa_{\alpha}, \varepsilon_{\mathrm{nl}}, \operatorname{grad}_{\mathrm{x}} \varepsilon_{\mathrm{nl}}\right\}
$$

where $\varepsilon_{\log }^{\mathrm{el}}$ and $\kappa_{\alpha}$ denote the elastic logarithmic strain and an arbitrary set of scalar internal variables, respectively. Additionally, the material's state is influenced by the micromorphic variable $\varepsilon_{\mathrm{nl}}$ and its spatial gradient.

\subsubsection{Balance Equations}

The necessary mechanical and micromorphic balance equations can be deduced with help of the principle of virtual power [23, 24]. The continuity equation (conservation of mass, mass density $\rho$ ) is assumed:

$$
\dot{\rho}+\rho \operatorname{div}_{\mathrm{x}} \vec{v}=0
$$

where $\vec{v}$ denotes the substantial velocity. In what follows, virtual quantities are highlighted by an asterisk ()* The internal virtual power $P_{\mathrm{i}}^{*}$ yields 


$$
P_{\mathrm{i}}^{*}=-\int_{\mathfrak{B}} p_{\mathrm{i}} \mathrm{d} v, \quad p_{\mathrm{i}}^{*}=\boldsymbol{\sigma}: \boldsymbol{D}^{*}+m_{\mathrm{nl}} \dot{\varepsilon}_{\mathrm{nl}}^{*}+\vec{M}_{\mathrm{nl}} \cdot \operatorname{grad}_{\mathrm{x}} \dot{\varepsilon}_{\mathrm{nl}}^{*}
$$

with the prescribed virtual power density $p_{\mathrm{i}}^{*}$. The spatial domain currently occupied by the material body is given by $\mathfrak{B}$. The boundary of the material body and the infinitesimal volume element are denoted as $\partial \mathfrak{B}$ and $d v$, respectively. The symmetric CAUCHY-stress tensor and the rate of deformation tensor are denoted as $\boldsymbol{\sigma}$ and $\boldsymbol{D}$, respectively. The virtual power is enriched by the micromorphic variable and its gradient as well as by their conjugated generalized stresses $m_{\mathrm{nl}}$ and $\vec{M}_{\mathrm{nl}}$.

The external virtual power is formulated by volume specific and contact terms

$$
P_{\mathrm{e}}^{*}=\int_{\mathfrak{B}} \rho \vec{f} \cdot \vec{v}^{*} \mathrm{~d} v+\int_{\partial \mathfrak{B}_{\mathrm{t}}} \vec{t} \cdot \vec{v}^{*} \mathrm{~d} a+\int_{\partial \mathfrak{B}_{\mathrm{m}}} m_{\mathrm{c}} \dot{\varepsilon}_{\mathrm{nl}}^{*} \mathrm{~d} a,
$$

where $\vec{f}$ is a mass specific force and $\vec{t}$ as well as $m_{\mathrm{c}}$ denote tractions acting on the infinitesimal surface element $\mathrm{d} a$.

Furthermore, a classical D'ALEMBERT inertia term is considered

$$
P_{\mathrm{a}}^{*}=-\int_{\mathfrak{B}} \rho \dot{\vec{v}} \cdot \vec{v}^{*} \mathrm{~d} v
$$

Evaluating the principle of virtual power,

$$
P_{\mathrm{i}}^{*}+P_{\mathrm{e}}^{*}+P_{\mathrm{a}}^{*}=0
$$

yields the following balance equations: Firstly, the balance of linear momentum is obtained, which is complemented by boundary and initial conditions:

$$
\begin{aligned}
\operatorname{div}_{\mathrm{x}} \sigma+\rho \vec{f} & =\rho \dot{\vec{v}} & \forall \vec{x} \in \mathfrak{B}, \\
\vec{t} & =\sigma \cdot \vec{n} & \forall \vec{x} \in \partial \mathfrak{B}_{\mathrm{t}} \\
\vec{u} & =\overline{\vec{u}} & \forall \vec{x} \in \partial \mathfrak{B}_{\mathrm{u}}, \\
\vec{v}\left(\vec{x}, t_{0}\right) & =\vec{v}_{0} & \forall \vec{x} \in \mathfrak{B} .
\end{aligned}
$$

The outward unit normal is introduced as $\vec{n}$.

Secondly, the micromorphic balance and related boundary conditions are obtained

$$
\begin{aligned}
\operatorname{div}_{\mathrm{x}} \vec{M}_{\mathrm{nl}} & =m_{\mathrm{nl}} & & \forall \vec{x} \in \mathfrak{B}, \\
m_{\mathrm{c}} & =\vec{M}_{\mathrm{nl}} \cdot \vec{n} & & \forall \vec{x} \in \partial \mathfrak{B}_{\mathrm{m}}, \\
\varepsilon_{\mathrm{nl}} & =\bar{\varepsilon}_{\mathrm{nl}} & & \forall \vec{x} \in \partial \mathfrak{B}_{\mathrm{e}} .
\end{aligned}
$$

Furthermore, the local forms of the thermodynamical principles read 


$$
\rho \dot{\psi}+\vartheta \dot{s}+s \dot{\vartheta}=p_{\mathrm{i}}-\operatorname{div}_{\mathrm{x}} \vec{q}_{\mathrm{th}}+\rho p_{\mathrm{th}} \quad \text { (energy balance) }
$$

and

$$
-\rho s \dot{\vartheta}-\rho \dot{\psi}+p_{\mathrm{i}}-\frac{1}{\vartheta} \vec{q}_{\mathrm{th}} \cdot \operatorname{grad}_{\mathrm{x}} \vartheta \geq 0 . \quad \text { (dissipation inequality). }
$$

Herein, the Helmholtz free energy $\psi$ is chosen as state potential. The specific entropy, the heat flux, and the mass specific heat source are denoted as $s, \vec{q}_{\text {th }}$, and $p_{\text {th }}$, respectively. The term $p_{\mathrm{i}}$ denotes the physical counterpart of the virtual power given in (22.4).

\subsubsection{Constitutive Assumptions and Equations}

The high formability and ductile damage mechanisms of TRIP-steels imply a finite deformation framework as basis of constitutive modeling. As constitutive assumption, an additive split of the rate of deformation tensor $\boldsymbol{D}$ into an elastic, viscoplastic, and transformation induced part is considered:

$$
\boldsymbol{D}=\boldsymbol{D}_{\mathrm{el}}+\boldsymbol{D}_{\mathrm{vpl}}+\boldsymbol{D}_{\text {trip }}
$$

A state potential based on the logarithmic strain is proposed:

$$
\psi=\psi\left(\varepsilon_{\log }^{\mathrm{el}}, \vartheta, \kappa_{\alpha}, \varepsilon_{\mathrm{nl}}, \operatorname{grad}_{\mathrm{x}} \varepsilon_{\mathrm{nl}}\right)
$$

The constitutive-dependent quantities $\left\{\sigma, s, K_{\alpha}, m_{\mathrm{nl}}, \vec{M}_{\mathrm{nl}}\right\}$ have the same dependencies as $\psi$ (principle of equi-presence). Utilizing the COLEMAN-NOLL-procedure [25], constitutive relations can be deduced from the dissipation inequality (22.16) plugging in the state potential (22.18):

$$
\boldsymbol{\sigma}=\rho \frac{\partial \psi}{\partial \boldsymbol{\varepsilon}_{\log }^{\mathrm{el}}}, s=-\frac{\partial \psi}{\partial \vartheta}, \vec{M}_{\mathrm{nl}}=\rho \frac{\partial \psi}{\partial \operatorname{grad}_{\mathrm{x}} \varepsilon_{\mathrm{nl}}}, m_{\mathrm{nl}}=\rho \frac{\partial \psi}{\partial \varepsilon_{\mathrm{nl}}} .
$$

The conjugated thermodynamic forces to the internal variables are defined as

$$
K_{\alpha}=\rho \frac{\partial \psi}{\partial \kappa_{\alpha}}
$$

Furthermore, expressions for the thermal and mechanical dissipation remain, which have to be fulfilled independently: 


$$
\begin{aligned}
& \gamma_{\text {th }}=-\frac{1}{\vartheta} \vec{q}_{\text {th }} \cdot \operatorname{grad}_{\mathrm{x}} \vartheta \geq 0, \\
& \gamma_{\mathrm{m}}=\sigma:\left(\boldsymbol{D}_{\mathrm{vpl}}+\boldsymbol{D}_{\text {trip }}\right)-K_{\alpha} \dot{\kappa}_{\alpha} \geq 0 .
\end{aligned}
$$

The thermal and mechanical dissipation inequalities set restrictions on the definitions of the heat flux vector $\vec{q}_{\text {th }}$, the evolution equations of the inelastic deformation rates $\left\{\boldsymbol{D}_{\mathrm{vpl}}, \boldsymbol{D}_{\text {trip }}\right\}$, and the internal variables $\kappa_{\alpha}$ to be proposed.

\subsubsection{State Potential}

To specify the state potential (22.18), the set of state variables is complimented by the choice of scalar internal variables $\kappa_{\alpha}=\left\{r, f_{\mathrm{sb}}, z, D\right\}$ :

- $r$ : strain hardening variable (isotropic)

- $f_{\mathrm{sb}}$ : volume fraction of shear bands

- $z$ : volume fraction of $\alpha^{\prime}$-martensite

- $D$ : damage variable (isotropic)

Subsequently, a multiplicative viscoplasticity approach is introduced, as classified by Lemaître and Chaboche [26, Sect. 6.4.2]. Therefore, the state potential must not contain the hardening variable $r$ to ensure non negative mechanical dissipation, see [26, Sect. 6.4.2]. Moreover, the stored energy in shear bands is neglected. Thus, the HELMHOLTZ free energy is given as $\psi\left(\varepsilon_{\log }^{\mathrm{el}}, \vartheta, z, D, \varepsilon_{\mathrm{nl}}, \operatorname{grad}_{\mathrm{x}} \varepsilon_{\mathrm{nl}}\right)$ :

$$
\rho \psi=\rho \psi_{\mathrm{el}}\left(\varepsilon_{\mathrm{log}}^{\mathrm{el}}, \vartheta, D\right)+\rho \psi_{\mathrm{chem}}(\vartheta, z)+\rho \psi_{\vartheta}(\vartheta)+\rho \psi_{\mathrm{nl}}\left(\varepsilon_{\mathrm{nl}}, \operatorname{grad}_{\mathrm{x}} \varepsilon_{\mathrm{nl}}\right)
$$

The thermoelastic part reads

$$
\psi_{\mathrm{el}}=(1-D) \frac{1}{\rho} \frac{1}{2}\left(\varepsilon_{\log }^{\mathrm{el}}-\alpha_{\mathrm{th}}\left(\vartheta-\vartheta_{0}\right) \delta\right): \mathcal{C}:\left(\varepsilon_{\log }^{\mathrm{el}}-\alpha_{\mathrm{th}}\left(\vartheta-\vartheta_{0}\right) \delta\right) .
$$

Isotropic thermoelastic properties are assumed for the cast TRIP-steel, defined by the fourth order tensor of elastic stiffness $\mathcal{C}$ and the thermal expansion coefficient $\alpha_{\text {th }}$. Moreover, the elastic and thermal expansion properties are assumed to be similar for the austenitic and martensitic phase [27]. Damage $D$ degrades the elastic energy leading to material softening.

The chemical part describes the difference in GIBBS-energy of the two phases given by a rule of mixture:

$$
\psi_{\text {chem }}=(1-z) \psi_{\mathrm{a}}(\vartheta)+z \psi_{\mathrm{m}}(\vartheta)
$$


The purely temperature dependent term

$$
\psi_{\vartheta}=c_{\epsilon}^{0}\left(\vartheta-\vartheta \ln \left(\frac{\vartheta}{\vartheta_{*}}\right)\right)
$$

defines a constant part $c_{\epsilon}^{0}$ of the specific heat similar for both phases with a reference temperature $\vartheta_{*}$.

The last term in (22.23) comprises the micromorphic contributions. A conventional quadratic structure is prescribed, see [23]:

$$
\begin{aligned}
\rho \psi_{\mathrm{nl}} & =\frac{1}{2} H_{\mathrm{nl}}\left(\int_{t_{0}}^{t} \dot{\varepsilon}_{1}\left(\boldsymbol{D}_{\mathrm{vpl}}\right) \mathrm{d} \tau-\varepsilon_{\mathrm{nl}}\right)^{2} \\
& +\frac{1}{2} A_{\mathrm{nl}} \operatorname{grad}_{\mathrm{x}} \varepsilon_{\mathrm{nl}} \cdot \operatorname{grad}_{\mathrm{x}} \varepsilon_{\mathrm{nl}} .
\end{aligned}
$$

The first part penalizes the difference of the local strain like value, defined by the integral, and the micromorphic variable $\varepsilon_{\mathrm{nl}}$. The penalty stiffness is prescribed by the parameter $H_{\mathrm{nl}}$. The integral defines a scalar value, which is extracted from the viscoplastic deformation rate $\boldsymbol{D}_{\mathrm{vpl}}$ serving as driving force for damage. The second term states the micromorphic gradient contribution weighted by the parameter $A_{\mathrm{nl}}$.

\subsubsection{Consistent Rate Formulation}

The CAUCHY-stress defined in (22.19) can be evaluated using (22.23) and (22.24):

$$
\boldsymbol{\sigma}=\rho \frac{\partial \psi}{\partial \boldsymbol{\varepsilon}_{\log }^{\mathrm{el}}}=(1-D) \mathcal{C}:\left(\boldsymbol{\varepsilon}_{\log }^{\mathrm{el}}-\alpha_{\mathrm{th}}\left(\vartheta-\vartheta_{0}\right) \boldsymbol{\delta}\right)
$$

Applying the effective stress concept [28], the constitutive equation reads

$$
\hat{\sigma}=\frac{\sigma}{1-D}=\mathcal{C}:\left(\varepsilon_{\log }^{\mathrm{el}}-\alpha_{\mathrm{th}}\left(\vartheta-\vartheta_{0}\right) \delta\right)
$$

where $\hat{\sigma}$ designates the effective stress acting on an undamaged cross section of the material; $\sigma$ is then interpreted as the net stress. In what follows, a hat $(\hat{.})$ highlights quantities which are computed from the effective stress tensor $\hat{\boldsymbol{\sigma}}$.

The stress-strain relation has to be given in an objective rate form in order to implement the model into the user subroutine UMAT of the FEM-software ABAQUS. As objective time derivative, the logarithmic rate $(\cdot)$ introduced by Xiao et al. $[29,30]$ is applied to both sides of (22.29). Hence, the rate formulation 


$$
\hat{\hat{\boldsymbol{\sigma}}}=\mathcal{C}:\left(\boldsymbol{D}_{\mathrm{el}}-\alpha_{\mathrm{inst}} \boldsymbol{\delta} \dot{\vartheta}\right),
$$

is consistent with the notion of elasticity [31], where $\alpha_{\text {inst }}$ denotes the instantaneous coefficient of thermal expansion. The logarithmic rate of the logarithmic strain tensor $\boldsymbol{\varepsilon}_{\log }^{\mathrm{el}}$ yields the rate of deformation tensor $\boldsymbol{D}_{\mathrm{el}}$ as shown in [30]. The logarithmic rate of the effective CAUCHY-stress reads

$$
\stackrel{\hat{\boldsymbol{\sigma}}}{=}=\frac{\mathrm{d} \hat{\boldsymbol{\sigma}}}{\mathrm{d} t}-\boldsymbol{\Omega}_{\log } \cdot \hat{\boldsymbol{\sigma}}-\hat{\boldsymbol{\sigma}} \cdot \boldsymbol{\Omega}_{\log }^{\mathrm{T}} .
$$

The skew logarithmic spin $\boldsymbol{\Omega}_{\log }$ can be found in [29].

\subsubsection{Generalized Stresses}

The CoLEMAN-relations for generalized stresses in (22.19) are specified as

$$
\begin{aligned}
& \vec{M}_{\mathrm{nl}}=A_{\mathrm{nl}} \operatorname{grad}_{\mathrm{x}} \varepsilon_{\mathrm{nl}}, \\
& m_{\mathrm{nl}}=H_{\mathrm{nl}}\left(\varepsilon_{\mathrm{nl}}-\int_{t_{0}}^{t} \dot{\varepsilon}_{1}\left(\boldsymbol{D}_{\mathrm{vpl}}\right) \mathrm{d} \tau\right)=H_{\mathrm{nl}}\left(\varepsilon_{\mathrm{nl}}-\varepsilon_{1}\right),
\end{aligned}
$$

where the integral is substituted for reasons of clarity by $\varepsilon_{1}$. With (22.32) and (22.33) at hand, the micromorphic balance (22.12) can be rewritten in the established manner $[23,32]$

$$
L_{\mathrm{nl}}^{2} \Delta_{\mathrm{x}} \varepsilon_{\mathrm{nl}}=\varepsilon_{\mathrm{nl}}-\varepsilon_{1} \quad \forall \vec{x} \in \mathfrak{B},
$$

where the internal length $L_{\mathrm{nl}}$ is defined as

$$
L_{\mathrm{nl}}^{2}=\frac{A_{\mathrm{nl}}}{H_{\mathrm{nl}}} \geq 0 .
$$

The spatial Laplacian is denoted as $\Delta_{\mathrm{x}}$. Thereby, the micromorphic balance (22.34) is identical to the partial differential equation of implicit gradient enhancement proposed by Peerlings et al. [33], which has been used in own preliminary studies [34, 35]. Following the suggestion in [33], trivial natural boundary conditions are prescribed on all free boundaries

$$
\operatorname{grad}_{\mathrm{x}} \varepsilon_{\mathrm{nl}} \cdot \vec{n}=0 \quad \forall \vec{x} \in \partial \mathfrak{B}_{\mathrm{m}}=\partial \mathfrak{B} .
$$

As result of this choice, the overall mean values of the micromorphic variable and its local counterpart are equal [34]. 


\subsubsection{Thermodynamic Forces}

The conjugated thermodynamic forces $K_{\alpha}=\left\{R, F_{\mathrm{sb}}, Z, Y\right\}$ associated with the set of internal variables are specified by $(22.20)$ as

$$
\begin{aligned}
R & =\rho \frac{\partial \psi}{\partial r}=0 \\
F_{\mathrm{sb}} & =\rho \frac{\partial \psi}{\partial f_{\mathrm{sb}}}=0 \\
Z & =\rho \frac{\partial \psi}{\partial z}=\rho \psi_{\mathrm{m}}(\vartheta)-\rho \psi_{\mathrm{a}}(\vartheta)=\Delta g_{\mathrm{chem}}^{\mathrm{a} \rightarrow \mathrm{m}}(\vartheta) \\
Y & =\rho \frac{\partial \psi}{\partial D}=-\frac{1}{2}\left(\varepsilon_{\log }^{\mathrm{el}}-\alpha_{\mathrm{th}}\left(\vartheta-\vartheta_{0}\right) \delta\right): \mathcal{C}:\left(\varepsilon_{\log }^{\mathrm{el}}-\alpha_{\mathrm{th}}\left(\vartheta-\vartheta_{0}\right) \delta\right)
\end{aligned}
$$

The driving force for martensitic phase transition $Z$ consists of the energy difference $\triangle g_{\text {chem }}^{\mathrm{a} \rightarrow \mathrm{m}}(\vartheta)$, which is negative once the temperature is below the thermodynamic equilibrium temperature [36], i.e., in the region of interest. The energy release rate $Y$ is always negative or zero, $Y \leq 0$.

\subsubsection{Dissipation and Heat Equation}

The mechanical dissipation (22.22) can be intermediately specified as

$$
\gamma_{\mathrm{m}}=\boldsymbol{\sigma}:\left(\boldsymbol{D}_{\mathrm{vpl}}+\boldsymbol{D}_{\text {trip }}\right)-Z \dot{z}-Y \dot{D}
$$

The requirement $\gamma_{\mathrm{m}} \geq 0$ is discussed in detail after introducing the evolution laws for the martensite volume fraction $\dot{z}$ and the damage variable $\dot{D}$ for the different modeling approaches.

The heat equation can be obtained from the energy balance (22.15), see [9]. Neglecting coupling terms and assuming FoURIERs law, a rudimentary format can be obtained typically used for computation purposes [9]:

$$
\rho c_{\epsilon}^{0} \dot{\vartheta}=\lambda_{\text {th }} \Delta_{\mathrm{x}} \vartheta+\tilde{p} \quad \forall \vec{x} \in \mathfrak{B}
$$

with the heat source

$$
\tilde{p}=\gamma_{\mathrm{m}}+\rho p_{\mathrm{th}}
$$

The specific heat $c_{\epsilon}^{0}$ and the conductivity $\lambda_{\text {th }}$ are assumed as constants. 


\subsection{Material Models}

\subsubsection{Preliminaries for both Models}

Both considered material models are based on the previously introduced thermomechanical framework and a multiplicative viscoplasticity formulation discussed within the next subsection. In order to describe the $\alpha^{\prime}$-martensite formation, two modifications of the OLSON-COHEN model [37] (OC-model) for strain induced martensite evolution are developed for thermomechanical loading conditions: A micromechanically extended approach and a simplified empirical model allowing for a fast parameter calibration. The macroscopic TRIP-kinematics are introduced to conclude the section.

\subsubsection{Multiplicative Viscoplasticity}

In order to take characteristic strain rate effects of high alloy TRIP-steels into account, a multiplicative viscoplasticity framework is utilized. A dissipation potential of NORTON-type is proposed:

$$
\phi(\sigma)=\frac{(1-D) \dot{\varepsilon}_{0} \sigma_{\mathrm{y}}}{m+1}\left(\frac{\hat{\sigma}_{\mathrm{eq}}}{\sigma_{\mathrm{y}}}\right)^{(m+1)} .
$$

The model parameters $m$ and $\dot{\varepsilon}_{0}$ control the strain rate sensitivity. Isotropic strain hardening can be taken into account by the yield stress $\sigma_{\mathrm{y}}(r, z)$. Within the next sections, the potential (22.44) is extended by contributions of all invariants of the effective stress tensor:

$$
\hat{I}_{1}=\operatorname{tr}(\hat{\boldsymbol{\sigma}}), \quad \hat{J}_{2}=\frac{1}{2} \hat{\boldsymbol{S}}: \hat{\boldsymbol{S}}, \quad \hat{J}_{3}=\frac{1}{3} \operatorname{tr}(\hat{\boldsymbol{S}} \cdot \hat{\boldsymbol{S}} \cdot \hat{\boldsymbol{S}}),
$$

where the stress deviator is introduced as

$$
\hat{\boldsymbol{S}}=\hat{\sigma}-\frac{1}{3} \hat{I}_{1} \boldsymbol{\delta}
$$

The unit tensor of second order is denoted as $\delta$. The original NORTON-potential (22.44) is only influenced by the VON MISES equivalent stress

$$
\hat{\sigma}_{\mathrm{eq}}=\sqrt{3 \hat{J}_{2}}
$$




\subsubsection{Strain Induced Martensite}

The well established OC-model [37] is based on the observation that nuclei of $\alpha^{\prime}$ martensite are predominantly formed at intersections of shear bands. The evolution of shear band volume fraction $f_{\mathrm{sb}}$ is modeled as

$$
\dot{f}_{\mathrm{sb}}=\alpha_{\mathrm{oc}}\left(1-f_{\mathrm{sb}}\right) \dot{\varepsilon}_{\mathrm{eq}},
$$

where the parameter $\alpha_{\mathrm{oc}}$ is a function of temperature and stress state [38-40]. As scalar measure of plastic deformation, the rate of equivalent (visco-)plastic deformation $\dot{\varepsilon}_{\text {eq }}$ is utilized. The original evolution equation of $\alpha^{\prime}$-martensite volume fraction $z$ proposed by Olson and Cohen [37] reads

$$
\dot{z}=(1-z) \beta_{\mathrm{oc}} n_{\mathrm{oc}} f_{\mathrm{sb}}^{\left(n_{\mathrm{oc}}-1\right)} \dot{f}_{\mathrm{sb}} .
$$

The probability to form a martensite embryo at a shear band intersection is cast into the parameter $\beta_{\mathrm{oc}}$. The specific geometric setting of the shear bands is taken into account by the parameter $n_{\mathrm{oc}}$. Modifications of (22.48) and (22.49) are discussed in the separate section of the different material models.

\subsubsection{TRIP Kinematics}

During martensitic phase transformation, inelastic strains occur which become visible on the macroscopic scale of polycrystalline material [41, 42]. These so-called TRIP-strains comprise a volumetric part and a deviatoric part. Two effects lead to these inelastic strains: A formation of favorable oriented martensite variants according to the applied stress state (MAGEE-effect) and additional plastic deformation of the austentite during accommodation of newly formed martensite (GREENWOODJOHNSON-effect), see [43] and [44, p. 69ff]. An empirical evolution law of the TRIPdeformation rate reads

$$
\boldsymbol{D}_{\text {trip }}=\left(k_{\mathrm{gj}} \hat{\sigma}_{\mathrm{eq}} \frac{\mathrm{d} \varphi(z)}{\mathrm{d} z}+k_{\mathrm{s}}\right) \boldsymbol{N} \dot{z}+\frac{1}{3} \triangle_{\mathrm{v}} \boldsymbol{\delta} \dot{z}
$$

with the flow normal

$$
\boldsymbol{N}=\frac{3}{2 \hat{\sigma}_{\mathrm{eq}}} \hat{\boldsymbol{S}}
$$

From (22.50), many modifications proposed in literature can be deduced [9, 12, 38, 39, 41, 45-50]. The first part shows that the magnitude of the TRIP-strain depends on the applied stress level as measured by Nagayama et al. [42]; the parameter $k_{\mathrm{gj}}$ is called GREENWOOD- JOHNSON-constant. The additional constant contribution $k_{\mathrm{s}}$ is used by Stringfellow et al. [38]. 
The volumetric strain is prescribed by the parameter $\triangle_{\mathrm{V}}$, which typically ranges from 2 to $4 \%$ depending on the carbon content of the considered alloy.

Nagayama et al. [42] and Ahrens [41] conducted experiments on temperature induced martensitic transformation and simultaneously applied external stresses. From these experiments, a magnitude for the stress dependent, deviatoric uni-axial TRIP-strain of about 1.5-2.0\% can be found. This is in accordance to the statement of Martin [51, Sect. 2.1.6] that the TRIP-contribution to the whole inelastic deformation is comparatively small. The high ductility of high alloy TRIP-steels is attributed to the excellent strain hardening, which postpones localization effects.

\subsubsection{Micromechanically Motivated Model}

Starting with the assumption that formed $\alpha^{\prime}$-martensite nuclei and domains act as inclusions, homogenization schemes can be developed to predict the effective macroscopic material response. Thereby, the modeling gains a microstructural wellfounded character. The utilized analytic homogenization approach is well documented in literature. Therefore, only a brief summary is given, which is needed to understand the results on crack tip loading in Sect.22.4.3. For details, the reader is referred to the numerous available publications devoted to the model development within CRC 799 [9, 22, 47, 52, 53]. Damage effects are neglected for this approach, i.e., $D=0$ and $\hat{\sigma}=\sigma$.

\subsubsection{Homogenization Method}

It is assumed that the viscoplastic properties of austenite and martensite differ widely. Therefore, independent dissipation potentials according to (22.44) are introduced for each phase. The rate of viscoplastic equivalent strain follows for each phase $l$ as

$$
\dot{\varepsilon}_{\mathrm{eq}}^{(l)}=\frac{\partial \phi^{(l)}\left(\sigma_{\mathrm{eq}}^{(l)}\right)}{\partial \sigma_{\mathrm{eq}}^{(l)}}=\dot{\varepsilon}_{0}\left(\frac{\sigma_{\mathrm{eq}}^{(l)}}{\sigma_{\mathrm{y}}^{(l)}}\right),
$$

where $l=\mathrm{a}$ and $l=\mathrm{m}$ denote austenite and martensite, respectively. The macroscopically effective properties are determined as described in [15, 22]. A variational principle is utilized, which is established by Ponte Castañeda and Suquet [54]. The necessary condition for an optimally chosen linear comparison composite is deduced in the case of statistically isotropic and uniformly distributed phases with isotropic behavior as

$$
\sigma_{\mathrm{eq}}^{(l)}=\sigma_{\mathrm{eq}} \sqrt{\frac{1}{v^{(l)}} \frac{\partial \Theta^{\mathrm{hom}}}{\partial \Theta^{(l)}}},
$$


where the effective compliance $\Theta^{\text {hom }}$ is computed using a lower Hashin-Shtrikman bound

$$
\Theta^{\text {hom }}=\frac{v^{\mathrm{m}}\left(\frac{2}{\Theta^{\mathrm{a}}}+\frac{3}{\Theta}\right)+v^{\mathrm{a}}\left(\frac{2}{\Theta^{\mathrm{m}}}+\frac{3}{\Theta}\right)}{\frac{v^{\mathrm{m}}}{\Theta^{\mathrm{m}}}\left(\frac{2}{\Theta^{\mathrm{a}}}+\frac{3}{\Theta}\right)+\frac{v^{\mathrm{a}}}{\Theta^{\mathrm{a}}}\left(\frac{2}{\Theta^{\mathrm{m}}}+\frac{3}{\Theta}\right)} .
$$

Therein, $v^{(l)}$ denotes the volume fraction of phase $l$, i.e., $v^{\mathrm{m}}=z$ and $v^{\mathrm{a}}=1-z$. The value of $\Theta$ is chosen as $\Theta=\max \left(\Theta^{\mathrm{a}}, \Theta^{\mathrm{m}}\right)$, where the compliance of a single phase is defined as

$$
\Theta^{(l)}=\frac{3}{\sigma_{\mathrm{eq}}^{(l)}} \dot{\varepsilon}_{\mathrm{eq}}^{(l)} .
$$

The strain hardening functions of each phase $\sigma_{\mathrm{y}}^{(l)}$ are chosen as power laws.

A stress state dependent yielding and strain hardening can be introduced by taking the third invariant of stress deviator $J_{3}$ into account. This leads to major modifications of the homogenization scheme. The reader is referred to the work of Prüger [22] dealing with this topic.

\subsubsection{Martensite Kinetics and TRIP-kinematics: Stringfellow-Model}

An established modification of the OC-model has been proposed by Stringfellow et al. [38]. Further enhancements have been incorporated, e.g., in [9, 39, 40]. Due to the homogenizations approach, plastic deformations of the single phases are accessible. Thus, the dependencies of the martensite kinetics on equivalent plastic strain are substituted as functions of the viscoplastic strain in austenite $\dot{\varepsilon}_{\text {eq }}^{\text {a }}$. The modified OC-kinetics of strain induced martensite, following [9], read as

$$
\dot{z}=(1-z)\left(A \dot{f}_{\mathrm{sb}}+B(\dot{g}-\dot{\bar{g}})\right)
$$

with prefactors

$$
\begin{aligned}
& A=\beta_{\mathrm{oc}} n_{\mathrm{oc}} f_{\mathrm{sb}}^{\left(n_{\mathrm{oc}}-1\right)} P, \\
& B=\beta_{\mathrm{oc}} f_{\mathrm{sb}}^{n_{\mathrm{oc}}} \frac{\mathrm{d} P}{\mathrm{~d} g} H(\dot{P}),
\end{aligned}
$$

and the normal distribution

$$
P=\frac{1}{\sqrt{2 \pi s_{\mathrm{g}}}} \int_{-\infty}^{g} \exp \left[-\frac{1}{2}\left(\frac{g^{*}-\bar{g}}{s_{\mathrm{g}}}\right)^{2}\right] \mathrm{d} g^{*} .
$$


Therein, $g$ denotes the driving force for martensite formation and $\bar{g}$ a barrier function [55]:

$$
\bar{g}=\bar{g}_{0}+\bar{g}_{1} \varepsilon_{\mathrm{eq}}^{\mathrm{a}}
$$

The driving force $g$ is formulated as the sum of the chemical contribution $Z(\vartheta)$ and a mechanical part motivated from the energy term $\sigma: \boldsymbol{D}_{\text {trip }}[22$, Sect. 5.4]:

$$
g=-Z+\left(\left(k_{\mathrm{gj}} \sigma_{\mathrm{eq}} \frac{\mathrm{d} \varphi(z)}{\mathrm{d} z}+k_{\mathrm{s}}\right) \sigma_{\mathrm{eq}}+\triangle_{\mathrm{v}} \sigma_{\mathrm{h}}\right) .
$$

The chemical driving force $Z$ is typically determined by thermodynamical calculations, see [47]. Moreover, $s_{\mathrm{g}}$ is a fitting parameter of the model.

As mentioned above, the parameter of shear band formation $\alpha_{\mathrm{oc}}$ is a function of temperature and stress state. Prüger [22] proposes the approach

$$
\alpha_{\mathrm{oc}}(h, \vartheta)=\left\langle\alpha_{1}+\alpha_{2} \vartheta+\alpha_{3} \vartheta^{2}-\alpha_{4} \arctan (h)\right\rangle,
$$

where the stress triaxiality is introduced as

$$
h=\frac{\sigma_{\mathrm{h}}}{\sigma_{\mathrm{eq}}}
$$

with the hydrostatic stress

$$
\sigma_{\mathrm{h}}=\frac{1}{3} I_{1}
$$

The MACAULY-brackets

$$
\langle x\rangle= \begin{cases}0 & x<0 \\ x & x \geq 0\end{cases}
$$

in (22.62) ensure a monotonically increasing shear band volume fraction.

For the micromechanical approach, the generally introduced TRIP-deformation rate $\boldsymbol{D}_{\text {trip }}$ defined in (22.50) is utilized.

\subsubsection{Phenomenological Model}

The phenomenological modeling strategy is developed as a robust engineering tool comprising characteristic aspects of the thermomechanical, stress state dependent and damage behavior of high alloy TRIP-steel. Finally, two variants of the model are implemented into a FE-code: The first variant contains all thermomechanical 
features, the second includes ductile damage within the micromorphic framework at isothermal conditions. A combination of both is subject of current work.

\subsubsection{Viscoplastic Flow Rules}

As already introduced in the state potential (22.24), damage degrades the elastic properties and thus the bearable stress, see (22.28). Ductile damage is microscopically caused by the nucleation, growth, and coalescence of microvoids. At high stress triaxialities, growth of voids is the dominant damage mechanism, which influences the plastic yielding and flow behavior $[56,57]$. In this regime, the impact of hydrostatic stresses on plastic flow needs to be taken into account. A heuristic modification of the NORTON-potential (22.44) through a contribution of the hydrostatic stress is proposed

$$
\phi(\sigma)=\frac{(1-D) \dot{\varepsilon}_{0} \sigma_{\mathrm{y}}}{m+1}\left(\frac{\hat{\sigma}_{\mathrm{eq}}^{2}+q_{1} D\left\langle\hat{\sigma}_{\mathrm{h}}\right\rangle^{2}}{\sigma_{\mathrm{y}}^{2}}\right)^{\left(\frac{m+1}{2}\right)}
$$

A similar elliptic yield function in the rate independent framework is utilized in the gradient-enhanced damage model of Seupel and Kuna [35]. Dissipation potentials of similar structure are likewise proposed in creep damage mechanics [58].

An associated viscoplastic flow is assumed

$$
\boldsymbol{D}_{\mathrm{vpl}}=\frac{\partial \phi}{\partial \boldsymbol{\sigma}}=\dot{\varepsilon}_{\mathrm{eq}} \boldsymbol{N}+\dot{\varepsilon}_{\mathrm{h}} \boldsymbol{\delta}
$$

which leads to deviatoric and volumetric viscoplastic strains. The prefactors in (22.67) are identified as equivalent and volumetric viscoplastic strain rates

$$
\begin{aligned}
& \dot{\varepsilon}_{\mathrm{eq}}=\dot{\varepsilon}_{0} \frac{\hat{\sigma}_{\mathrm{eq}}}{\sigma_{\mathrm{y}}}\left(\frac{\hat{\sigma}_{\mathrm{eq}}^{2}+q_{1} D\left\langle\hat{\sigma}_{\mathrm{h}}\right\rangle^{2}}{\sigma_{\mathrm{y}}^{2}}\right)^{\left(\frac{m-1}{2}\right)}, \\
& \dot{\varepsilon}_{\mathrm{h}}=\dot{\varepsilon}_{0} \frac{\left\langle\hat{\sigma}_{\mathrm{h}}\right\rangle q_{1} D}{3 \sigma_{\mathrm{y}}}\left(\frac{\hat{\sigma}_{\mathrm{eq}}^{2}+q_{1} D\left\langle\hat{\sigma}_{\mathrm{h}}\right\rangle^{2}}{\sigma_{\mathrm{y}}^{2}}\right)^{\left(\frac{m-1}{2}\right)},
\end{aligned}
$$

respectively. The model parameter $q_{1}$ controls the hydrostatic stress dependency. As long as $q_{1}$ is set to zero or no damage occurs $D=0$, the original volume preserving NORTON-type flow is obtained. A relation between (22.68) and (22.69) can be found [59]:

$$
\dot{\varepsilon}_{\mathrm{h}}=\frac{q_{1} D\langle h\rangle}{3} \dot{\varepsilon}_{\mathrm{eq}}
$$


In presence of damage, the hydrostatic plastic flow linearly depends on stress triaxiality $h$. This is in accordance to micromechanical findings within creep damage mechanics [60], but contrary to the known exponential influence deduced in $[56,57]$, cf. [61]. In contrast to the GURSON-model, the volumetric plastic strain of the present model can just increase, because a volumetric flow occurs only for positive hydrostatic stresses.

An additional influence of the third stress invariant $\hat{J}_{3}$ on plastic flow is neglected, which is in accordance with investigations on initial yielding of the considered TRIPsteel conducted by Kulawinski et al. [62]. The asymmetric strain hardening is fully captured by the definition of the evolution equation for the hardening variable $r$.

\subsubsection{Isotropic Strain Hardening}

An empirical mixture rule is adopted in order to take the influence of formed $\alpha^{\prime}$ martensite on strain hardening into account, which has been successfully applied in other studies [12, 27, 50]. Thus, the yield stress reads

$$
\sigma_{\mathrm{y}}(r, z, \vartheta)=\sigma_{0}(\vartheta)+Z_{1} m_{\mathrm{z}}(z)+H(r)
$$

Furthermore, only the initial yield stress $\sigma_{0}$ is considered as function of temperature as employed for an AISI 304 steel elsewhere [27]. The additional hardening contribution of martensite is captured by the second term, where $m_{\mathrm{z}}(z)$ is introduced to modify the shape of the flow curve during martensite evolution. The choice of the hardening function ensures that no strain softening can occur during martensite formation for $\vartheta=$ const. Material degradation arises only due to the evolution of the damage variable $D$.

Because of the direct influence of the stress state dependent martensitic phase transition, asymmetric strain hardening in uni-axial tension and compression is naturally predicted by the model. Hence, a higher strain hardening would be predicted in tension than in compression due to the enhanced phase transformation in tension, whereas the inverse trend is experimentally observed [12], [63, Sect. 5.3.2]. To take the realistic asymmetric strain hardening into account, Seupel and Kuna [12] propose an evolution of the hardening variable $r$ depending on the orientation in stress space, which is described by the LODE-angle

$$
\cos 3 \phi=\frac{3 \sqrt{3}}{2} \frac{\hat{J}_{3}}{\hat{J}_{2}^{\frac{3}{2}}} .
$$

The evolution of the hardening variable reads

$$
\dot{r}=\left(1+\frac{b}{2}(1-\cos 3 \phi)\right) \dot{\varepsilon}_{\mathrm{eq}} \geq 0,
$$


where the parameter $b>-1$ controls the influence of stress state. For $b=0$, the hardening variable coincides with the equivalent viscoplastic strain. For $b>0$, the hardening rate is increased in case of uni-axial compression $(\cos 3 \phi=-1)$ compared to uni-axial tension $(\cos 3 \phi=1)$, which is in accordance with the experimentally revealed behavior.

The same tendencies in strain hardening are observed, if the TWIP-mechanism is active $[11,63]$. The tension-compression asymmetry vanishes, if planar dislocation glide is the dominant deformation mechanism, see [63, Sect. 5.3.2]. For the considered steel, the transition of deformation mechanisms is explained as function of temperature $[6,51]$. Accordingly, the hardening asymmetry should depend on deformation mechanism and/or temperature, respectively. Therefore, the parameter $b$ is assumed as function of martensite volume fraction and temperature in the following manner:

$$
b(z, \vartheta)= \begin{cases}b_{0} \exp \left(-\left(\frac{\vartheta-T_{0}}{T_{1}}\right)^{2}\right) \exp \left(-\left(\frac{z-z_{0}}{z_{1}}\right)^{2}\right) & \vartheta \geq T_{0} \\ b_{0} \exp \left(-\left(\frac{z-z_{0}}{z_{1}}\right)^{2}\right) & \vartheta<T_{0}\end{cases}
$$

Thereby, the asymmetric hardening asymptotically vanishes for temperatures above $T_{0}$. When martensite transformation is active, the asymmetry can additionally vary during loading at constant temperature due to the second exponential function, which contains the fitting parameters $z_{0}$ and $z_{1}$.

\subsubsection{Martensite Kinetics and TRIP-Kinematics: Empirical Model}

Motivated by the sound investigations on stress state dependency of strain induced martensite formation and the suggested empirical kinetics approach of Beese and Mohr [64], a less sophisticated, empirical extension of the OC-kinetics can be proposed. The original evolution equation introduced by Olson and Cohen [37] is considered, see (22.49). In $\beta_{\mathrm{oc}}$, the probability that a shear band intersection becomes a martensite embryo is included. As shown in [37], the parameters $\alpha_{\mathrm{oc}}$ and $\beta_{\mathrm{oc}}$ are functions of temperature. Similar to the proposal in (22.62) for the parameter of shear band formation, Seupel and Kuna [12] use

$$
\alpha_{\mathrm{oc}}(h, \vartheta)=\alpha_{\mathrm{t}}(\vartheta)\left[\alpha_{0}+\alpha_{1}\left(\frac{2}{\pi} \arctan \left(\alpha_{2} h\right)+1\right)\right] .
$$

As discussed in [9], the non-linear function of stress triaxiality should saturate to a certain level. As long as $\alpha_{1} \geq 0$, no negative shear band rate can be caused by the stress state. Regarding $\alpha_{1}<0$, a non-negative rate of shear bands is obtained, if the parameters fulfill $\alpha_{0} \geq 0$ and $\alpha_{1} \geq-\frac{\alpha_{0}}{2}$. As temperature influence, probability functions are chosen [37]: 


$$
\alpha_{\mathrm{t}}(\vartheta)= \begin{cases}\exp \left(-\left(\frac{\vartheta-\alpha_{3}}{\alpha_{4}}\right)^{2}\right) & \vartheta \geq \alpha_{3} \\ 1 & \vartheta<\alpha_{3}\end{cases}
$$

For $\beta_{\text {oc }}$, a similar structure given by (22.75) and (22.76) is assumed with parameters $\beta_{0}-\beta_{4}$. Despite the empirical character of the extended kinetics law, less parameters are needed compared to the enhanced STRINGFELLOW-approach (Sect. 22.3.2.2).

The generally introduced TRIP-deformation rate (22.50) is utilized with the following parameters:

$$
k_{\mathrm{gj}}=8.1 \times 10^{-5} \mathrm{MPa}^{-1}, \quad \varphi(z)=(2-z) z, \quad k_{\mathrm{s}}=0, \quad \triangle_{\mathrm{v}}=0.02 .
$$

This set of parameters leads to a comparatively small contribution of transformation induced plasticity to the whole deformation.

\subsubsection{Ductile Damage}

Damage models devoted to high alloy TRIP-steels have been mainly developed for applications at cryogenic temperatures employing concepts of continuum damage mechanics [65-67]. A ductile damage mechanism is assumed as common feature of the mentioned approaches. Regularized damage models for TRIP-steels are less investigated, except first studies [12].

The evolution of ductile damage is known to be dependent on plastic deformation and stress state. Seupel and Kuna [35] propose an empirical, regularized model for ductile damage, which is able to predict failure due to void based mechanisms at high stress triaxialities and shear dominated failure. The mentioned model is a gradientenhanced version of local engineering approaches, e.g., [68]. In the present section, the approach of Seupel and Kuna [35] is adopted for the micromorphic-viscoplastic framework.

The empirical approach to ductile failure is based on the hypothesis, that damage can be neglected in a certain deformation range, but evolves rather fast after initiation [68]. The initiation of damage is captured by a loading history dependent indicator function $\omega\left(\varepsilon_{\text {eq }}, \sigma\right)$, which is not directly coupled to the constitutive equations similar to well known failure criteria. Exceeding a critical value $\omega \geq \omega_{\mathrm{c}}$ determines the initiation of damage, i.e., the onset of evolution of the local damage driving force $\dot{\varepsilon}_{1}\left(\boldsymbol{D}_{\mathrm{vpl}}\right)$. Its evolution is proposed as

$$
\dot{\varepsilon}_{1}= \begin{cases}0, & \omega\left(\sigma, \varepsilon_{\mathrm{eq}}, z\right)<\omega_{\mathrm{c}} \\ q_{2} \dot{\varepsilon}_{\mathrm{eq}}+q_{3} \dot{\varepsilon}_{\mathrm{h}} \stackrel{(22.70)}{=}\left(q_{2}+\frac{q_{1} q_{3} D\langle h\rangle}{3}\right) \dot{\varepsilon}_{\mathrm{eq}}, & \omega\left(\sigma, \varepsilon_{\mathrm{eq}}, z\right) \geq \omega_{\mathrm{c}}\end{cases}
$$

The model parameters $q_{2}$ and $q_{3}$ can be adjusted to weight between shear $\left(\dot{\varepsilon}_{\text {eq }}\right)$ and void growth dominated damage $\left(\dot{\varepsilon}_{\mathrm{h}}\right)$, respectively. It should be mentioned, that the local damage driving strain $\varepsilon_{1}$ is associated to the dissipation potential and viscoplas- 
tic flow. Mediavilla et al. [69] propose a non-local damage model using a comparable driving strain, but therein the plastic yielding stays purely deviatoric.

The micromorphic counterpart $\varepsilon_{\mathrm{nl}}$ of the damage driving strain is given by solving the micromorphic balance (22.34). To reach at a regularized damage model, the damage evolution is formulated with help of the micromorphic (non-local) variable $\varepsilon_{\mathrm{nl}}$. Following [69], an auxiliary variable $\kappa$ is defined to avoid pathological healing effects, cf. [34, 35],

$$
\dot{\kappa} \geq 0, \quad \dot{\kappa}\left(\varepsilon_{\mathrm{nl}}-\kappa\right)=0, \quad \varepsilon_{\mathrm{nl}}-\kappa \leq 0 .
$$

An exponential dependency of damage evolution on $\kappa$ is assumed:

$$
\dot{D}= \begin{cases}3(1-D) \frac{\dot{\kappa}}{\varepsilon_{\mathrm{c}}} & D<D_{\mathrm{c}} \\ D_{\max } a^{*} \exp \left(-a^{*}\left(\kappa-\kappa^{*}\right)\right) \dot{\kappa} & D \geq D_{\mathrm{c}}\end{cases}
$$

or in integrated form

$$
D= \begin{cases}1-\left(1-D_{0}\right) \exp \left(-3 \frac{\kappa}{\varepsilon_{\mathrm{c}}}\right) & D<D_{\mathrm{c}} \\ D_{\max }\left(1-\exp \left(-a^{*}\left(\kappa-\kappa^{*}\right)\right)\right) & D \geq D_{\mathrm{c}}\end{cases}
$$

with

$$
\begin{aligned}
& a^{*}=\frac{3}{\varepsilon_{\mathrm{c}}} \frac{\left(1-D_{\mathrm{c}}\right)}{\left(D_{\max }-D_{\mathrm{c}}\right)}, \\
& \kappa^{*}=-\frac{\varepsilon_{\mathrm{c}}}{3} \ln \left(\frac{1-D_{\mathrm{c}}}{1-D_{0}}\right)+\frac{1}{a^{*}} \ln \left(1-\frac{D_{\mathrm{c}}}{D_{\max }}\right) .
\end{aligned}
$$

Because $\kappa$ is a monotonically increasing variable without upper limit, a totally damaged state $(D \approx 1)$ can be reached asymptotically. In order to ensure the robustness of the model near to total failure, the numerical value of damage is limited by a prescribed saturation of $D$ towards $D_{\max }<1$ as proposed by Seupel and Kuna [35] (second case in (22.80) and (22.81), respectively).

The exponential damage law is motivated by the evolution of porosity assuming a nearly volume preserving matrix material, c.f. [57]. The purely void growth based damage mechanism is included in the current formulation by imposing $\varepsilon_{\mathrm{c}}=1, q_{2}=0$ and $q_{3}=1$ with $D_{0}>0$. The additional parameter $\varepsilon_{\mathrm{c}}$ can be used to adjust the slope of the damage law. The saturation value of damage is prescribed as $D_{\max }=0.999 \approx 1$ and the transition value is set to $D_{\mathrm{c}}=0.995$, i.e., near the state of total material failure.

To avoid a spurious widening of the total damaged zone, Seupel and Kuna [35] suggest to fix the local contribution $\dot{\varepsilon}_{1}=0$ in case the totally damaged state $D \geq D_{\text {c }}$ is reached. This patch solution damps the widening of the totally damaged domain, but other sophisticated methods, cf. [70], should be taken into account in ongoing 
works. The zone of totally damaged integration points or finite elements mimics the actually discontinuous crack.

\subsubsection{Thermodynamic Consistency}

The mechanical dissipation introduced in (22.41) can be specified for the phenomenological model as

$$
\gamma_{\mathrm{m}}=\sigma_{\mathrm{eq}}\left(\dot{\varepsilon}_{\mathrm{eq}}+\hat{\sigma}_{\mathrm{eq}} k_{\mathrm{gj}} \frac{\mathrm{d} \varphi(z)}{\mathrm{d} z} \dot{z}\right)+\sigma_{\mathrm{h}}\left(\dot{\varepsilon}_{\mathrm{h}}+\triangle_{\mathrm{v}} \dot{z}\right)-\Delta g_{\text {chem }}^{\mathrm{a} \rightarrow \mathrm{m}} \dot{z}-Y \dot{D}
$$

The rates of the introduced internal variables are constructed to yield $\dot{\kappa}_{\alpha} \geq 0$. The first part of $\gamma_{\mathrm{m}}\left(\sigma_{\mathrm{eq}}(\ldots)\right)$ is always larger or equal to zero. The damage contribution is likewise non-negative, because $Y \leq 0$. The chemical contribution is positive as long as the temperature is below the thermodynamic equilibrium temperature. For temperatures above, this restriction is not fulfilled automatically and this part has to be controlled. For experimentally calibrated models, $\dot{z}$ should vanish in this temperature region, i.e., the chemical part is typically thermodynamically consistent. The dissipation contribution of volumetric plastic strain is always non-negative, see (22.69), whereas the volumetric TRIP-strains can cause a negative dissipation in compression states [9], i.e., if $\sigma_{\mathrm{h}}<0$. To ensure positive dissipation, the hydrostatic TRIP-part is neglected in the mechanical dissipation as proposed by Mahnken and Schneidt [46]. Nonetheless, it should be mentioned that the damage model without martensite evolution is thermodynamically consistent in a strict sense.

\subsubsection{Numerical Implementation}

In order to analyze complex boundary value problems numerically, the material models are implemented into the FE-code ABAQUS via the user-defined subroutine UMAT. Implementation aspects of the micromechanically motivated and phenomenological model within the thermomechanical framework are discussed in literature $[22,34,35]$. One particular aspect should be emphasized: The implementation of the micromorphic damage model is simplified due to the similarity of the HELMHOLTZ-type equation (22.34) and the steady state case of the heat equation (22.42) as pointed out in [34]. A simple renaming of variables $\left(\varepsilon_{\mathrm{nl}} \leftrightarrow \vartheta\right)$, a modification of the heat source $\left(\tilde{p} \leftrightarrow-\varepsilon_{\mathrm{nl}}+\varepsilon_{1}\right)$, and the interpretation of the conductivity $\lambda_{\text {th }} \leftrightarrow L_{\mathrm{nl}}^{2}$ allow a fast implementation into the commercial FE-code. A major advantage is the usage of the available thermomechanical finite element library and post-processing tools, which becomes interesting for engineering applications. 


\subsection{Results}

In this section the introduced models are applied. A detailed study on the phenomenological modeling approach from Sect. 22.3.3 is conducted to demonstrate its ability to comprise the influence of stress state and temperature on martensite kinetics as well as strain hardening behavior. Furthermore, the micromechanically motivated model from Sect. 22.3.2 is used in order to study the stress field in front of a crack. Additionally, the crack driving force is formulated in consideration of plastic deformation and phase transformation. Finally, a cohesive zone approach and the micromorphic damage model (Sect. 22.3.3) are utilized to simulate ductile fracture in a particular TRIP-steel.

\subsubsection{Material}

Consistent experimental investigations on a cast TRIP-steel X3CrMnNi 16-6-6 (see $[53,63])$ are used as reference for calibration and validation purposes. The exact chemical composition is given in Table 22.1. A detailed characterization of the material can be found in [63]. The material used to manufacture the CT-specimen for fracture mechanics test exhibits a slightly deviating composition, see Table 22.1.

\subsubsection{Deformation and Phase Transition Behavior}

For the phenomenological model from Sect. 22.3.3, a step-by-step calibration strategy is pursued: Firstly, the model parameters of the martensite kinetics law are estimated with help of experimental data. Afterwards, the temperature and stress state dependent isotropic strain hardening law is fitted to a characteristic subset of tests. Following Prüger et al. [9], a simultaneous calibration to uni-axial tests at quasi-static loading, but different temperatures can be used as starting point. Damage effects are neglected at this stage of modeling. The validity of the model is assessed by predictions of the material behavior at other temperature conditions, strain rates, and inhomogeneous loading states. A comparable assessment for the micromechanically motivated model discussed in Sect.22.3.2 can be found in recent literature [9, 22].

Table 22.1 Chemical composition of considered cast TRIP-steels in mass \%

\begin{tabular}{l|l|l|l|l|l|l|l}
\hline Alloy & $\mathrm{Fe}$ & $\mathrm{C}$ & $\mathrm{N}$ & $\mathrm{Cr}$ & $\mathrm{Mn}$ & $\mathrm{Ni}$ & $\mathrm{Si}$ \\
\hline X2CrMnNi 15-5-7 & Bal. & 0.03 & 0.03 & 15.5 & 6.1 & 6.1 & 0.9 \\
\hline X2CrNiMn 15-7-5 & Bal. & 0.023 & 0.086 & 14.5 & 5.36 & 6.85 & 0.88 \\
\hline
\end{tabular}


Fig. 22.1 Martensite volume fraction $z$ versus true strain $\epsilon$ curves for uni-axial tension-compression loadings at different temperatures and constant strain rate $\dot{\epsilon}_{\mathrm{t}}=4 \times 10^{-4} \mathrm{~s}^{-1}$ : symbols correspond to experimental data of cast

X3CrMnNi 16-6-6, solid lines correspond to the fitted empirical model

(Sect. 22.3.3.3)

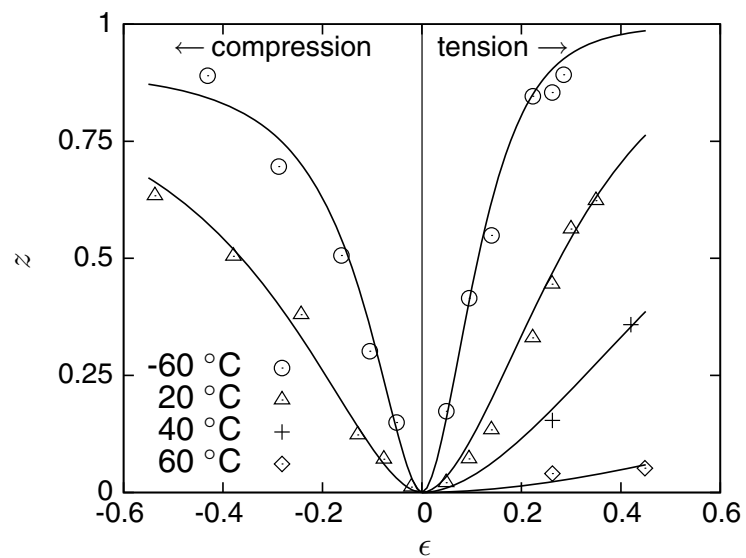

\subsubsection{Martensite Kinetics}

The empirical model of strain induced martensite kinetics (Sect.22.3.3.3) is calibrated using experimental data from uni-axial, quasi-static tensile and compression tests at different temperatures ranging from -60 to $60{ }^{\circ} \mathrm{C}[6,10,63,71]$. The calibration result is shown in Fig. 22.1 in terms of evolving martensite volume fraction during straining. A good match of the martensite evolutions is obtained with the a priori estimation of the model parameters given in Table 22.2 and the exponent $n_{\mathrm{oc}}=1.868$. For calibration purposes, the approximate coincidence of true strain $\epsilon$ and the equivalent plastic strain $\varepsilon_{\text {eq }}$ is assumed. Due to the occurrence of additional TRIP-strains depending on stress, a small deviation is to be expected finally.

\subsubsection{Asymmetric Strain Hardening}

The isotropic strain hardening contributions in (22.71) are chosen as functions of the hardening variable $r$, the temperature $\vartheta$, and the martensite volume fraction $z$ in the following manner [34]:

Table 22.2 Model parameters of empirical martensite kinetics (22.76)

\begin{tabular}{l|l|l|l|l|l|l|l|l|l}
\hline$\alpha_{0}$ & $\alpha_{1}$ & $\alpha_{2}$ & $\alpha_{3}$ & $\alpha_{4}$ & $\beta_{0}$ & $\beta_{1}$ & $\beta_{2}$ & $\beta_{3}$ & $\beta_{4}$ \\
\hline- & - & - & $\mathrm{K}$ & $\mathrm{K}$ & - & - & - & $\mathrm{K}$ & $\mathrm{K}$ \\
\hline 2.77 & 1.14 & -100 & 233.15 & 63.26 & 2.27 & 2.88 & 100 & 313.15 & 27.36 \\
\hline
\end{tabular}


Table 22.3 Model parameters of isotropic strain hardening (22.85)-(22.87)

\begin{tabular}{l|l|l|l|l|l|l|l|l}
\hline$\sigma_{0}$ & $H_{0}$ & $q$ & $H_{\text {inf }}$ & $r_{\mathrm{c}}$ & $Z_{1}$ & $Z_{2}$ & $c_{1}$ & $c_{2}$ \\
\hline $\mathrm{MPa}$ & $\mathrm{MPa}$ & - & $\mathrm{MPa}$ & - & $\mathrm{MPa}$ & - & - & $\mathrm{K}$ \\
\hline 174.2 & 1305.6 & 0.927 & 269.0 & 0.277 & 510.1 & 1.193 & -0.0014 & 373.15 \\
\hline
\end{tabular}

Table 22.4 Model parameters of asymmetric strain hardening (22.74)

\begin{tabular}{l|l|l|l|l}
\hline$b_{0}$ & $T_{0}$ & $T_{1}$ & $z_{0}$ & $z_{1}$ \\
\hline- & $\mathrm{K}$ & $\mathrm{K}$ & - & - \\
\hline 2.259 & 228.431 & 90.441 & 0.276 & 0.314 \\
\hline
\end{tabular}

$$
\begin{aligned}
H(r) & =\left\{\begin{array}{ll}
H_{0} r^{q} & r \leq r_{\mathrm{c}} \\
H_{0} r_{\mathrm{c}}^{q}+H_{\mathrm{inf}}\left(1-\exp \left(-\frac{H_{0} q r_{\mathrm{c}}^{q-1}}{H_{\mathrm{inf}}}\left(r-r_{\mathrm{c}}\right)\right)\right) & \text { else }
\end{array},\right. \\
\sigma_{0}(\vartheta) & =\exp \left(c_{1}\left(\vartheta-c_{2}\right)\right) \sigma_{0}, \\
m_{\mathrm{z}}(z) & =1-\exp \left(-Z_{2} z\right) .
\end{aligned}
$$

Thermoelastic properties for the considered steel are taken from literature [9]. Appropriate values for the parameters of strain rate sensitivity $m$ and $\dot{\varepsilon}_{0}$ are also proposed in literature [9, 48]: $m=40$ and $\dot{\varepsilon}_{0}=4 \times 10^{-4} \mathrm{~s}^{-1}$. Nine model parameters for isotropic strain hardening are to be identified and calibrated (22.85)-(22.87). Additionally, five parameters for stress state dependent evolution of the hardening variable have to be estimated, see (22.74). The final sets of estimated parameters are summarized in Tables 22.3 and 22.4.

As reference test data, force versus elongation curves of uni-axial, quasi-static tension and compression tests at $-60,20$ and $100{ }^{\circ} \mathrm{C}[6,10,63,71]$ are chosen. At these temperatures, different amounts of martensite are reached, from nearly $100 \%$ down to $0 \%$, see Fig. 22.1. The reasonable calibration results are illustrated in Fig. 22.2.

The stress state dependent strain hardening becomes clearly visible in terms of the true stress versus strain curves, see Fig. 22.3. In addition to the calibrated temperatures, predictions are made for 60 and $200{ }^{\circ} \mathrm{C}$. The corresponding experimental data is published in $[6,10,63,71]$. From -60 to $60^{\circ} \mathrm{C}$, substantial higher stress levels are attained in uni-axial compression compared to tension. The asymmetry vanishes at about $200{ }^{\circ} \mathrm{C}$, because neither martensite nor shear bands are formed, which is predicted by the model. Moreover, the qualitative change in strain hardening with temperature is reflected. The prediction at $60{ }^{\circ} \mathrm{C}$ in the compression regime deviates slightly. This indicates that other effects, especially the TWIP-effect, and their influence on strain hardening have to be considered in ongoing works. 

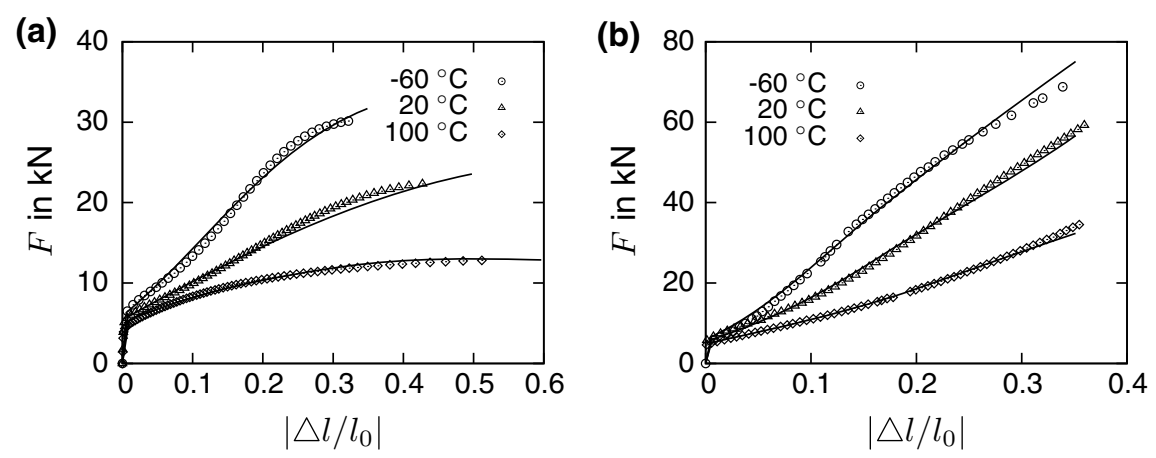

Fig. 22.2 Force $F$ versus elongation $\Delta l / l_{0}$ curves at different temperatures and constant engineering strain rate $\dot{\epsilon}_{\mathrm{t}}=4 \times 10^{-4} \mathrm{~s}^{-1}$, symbols correspond to experimental data of cast $\mathrm{X} 3 \mathrm{CrMnNi}$ 16-6-6, solid lines correspond to the fitted phenomenological model, a Uni-axial tension, b Uni-axial compression
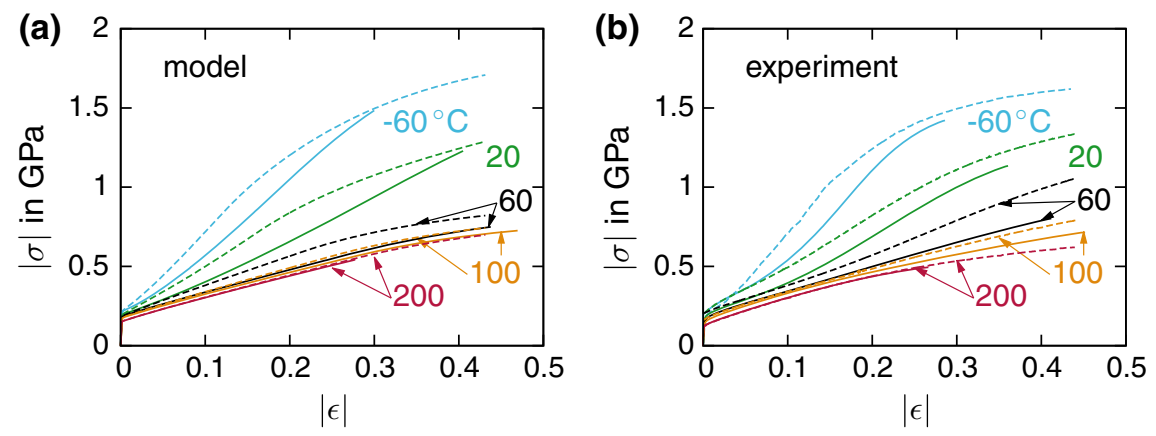

Fig. 22.3 True stress $\sigma$ versus true strain $\epsilon$ curves for different temperatures and stress states at $\dot{\epsilon}_{\mathrm{t}}=4 \times 10^{-4} \mathrm{~s}^{-1}$, solid lines correspond to tensile and dashed lines to compressive loadings, respectively; a Model predictions, b Experimental data of cast X3CrMnNi 16-6-6

\subsubsection{Strain Rate Dependency}

A characteristic strain rate effect of high alloy TRIP-steels is the so-called curve crossing observed in tensile tests [9]: The temperature increase during plastic deformation inhibits the formation of $\alpha^{\prime}$-martensite and thereby restricts the strain hardening capability. The temperature increases with increasing strain rates, because adiabatic conditions are asymptotically reached. The typical experimental observation based on data from [10, 63, 71, 72] is shown in Fig. 22.4: After a higher stress level at low strains, the curves of higher strain rates drop below the quasi-static reference curve. In order to reproduce this behavior, a thermomechanical coupling is mandatory [9].

For the numerical simulations via FEM, a FE-implementation of the whole experimental setup becomes necessary in order to consider the heat transfer from the specimen to clamps and environment. A detailed description of the utilized 
Fig. 22.4 Stress $\sigma$ and martensite volume fraction $z$ versus true strain $\epsilon$ curves at different technical strain rates $\dot{\epsilon}_{\mathrm{t}}$ during uni-axial tension test: symbols correspond to experimental data of cast X3CrMnNi 16-6-6, solid lines denote the model prediction

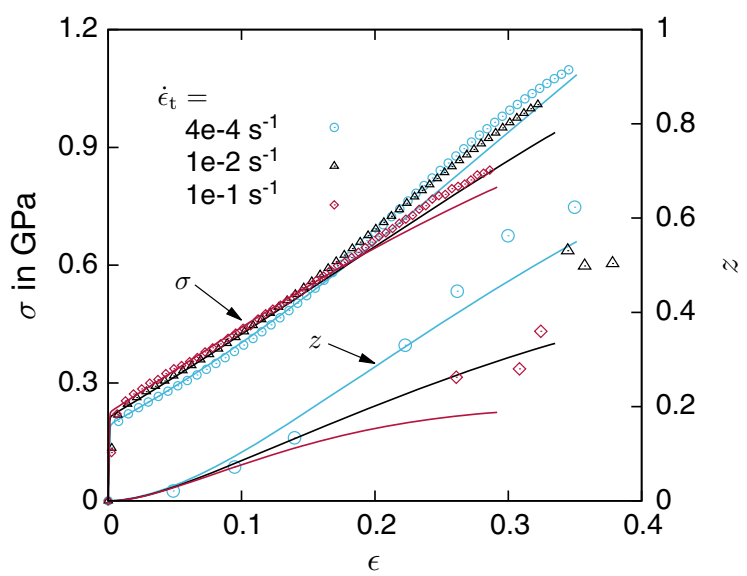

FE-model is given in [9]. For the micromechanically motivated model introduced in Sect.22.3.2, the ability to cover the curve crossing effect is shown in [9]. The same analysis is conducted for the phenomenological model with the results summarized in Fig. 22.4: Qualitatively, the curve crossing in strain hardening and the lower $\alpha^{\prime}$-martensite content at higher strain rates can be reproduced. A good match of the experimental stress versus strain curves is visible for strains $\epsilon<0.18$ for all considered strain rates. The predictions cannot fit for higher strain rates exactly, because there is an initial deviation of the quasi-static case at strains larger than 0.18. Additionally, due to the full dissipative formulation of the model, a temperature increase $(\Delta \vartheta \approx 70 \mathrm{~K})$ is predicted which is considerably higher than measured in experiments $(\Delta \vartheta \approx 45 \mathrm{~K},[63])$. As result, the strain hardening and martensite formation are weakened too much. This can be adjusted by introducing an empirical TAYLORQUINNEY-coefficient controlling the amount of dissipated power, see [73]. Despite the discussed need of improvements, the predictions of the models are physically reasonable.

\subsubsection{Inhomogeneous Loading States}

In addition to the uni-axial tests from [63], a series of round notched tensile tests has been conducted [53]. As test conditions, room temperature $\left(20^{\circ} \mathrm{C}\right)$ and quasistatic loading has been applied. A detailed description of the test setup and the evaluation procedure are given in [53]. The predictions of the calibrated material model are assessed by a comparison with the experimental results in Fig. 22.5. Good predictions of the force response are nearly made up to the onset of failure indicated by a rather sudden load drop for all differently notched specimens. Due to neglected damage, clear overestimations in the post-failure regime are visible. The indicated softening within the simulated curves is purely due to necking. Material softening caused by damage is taken into account in Sect.22.4.4.2. 
Fig. 22.5 Force $F$ versus elongation $\Delta l / l_{0}$ curves for differently notched tensile tests, numbers highlight the notch radius in $\mathrm{mm}$ (RS—-smooth test); solid lines correspond to the model prediction, dashed lines denote experiments of cast X3CrMnNi 16-6-6

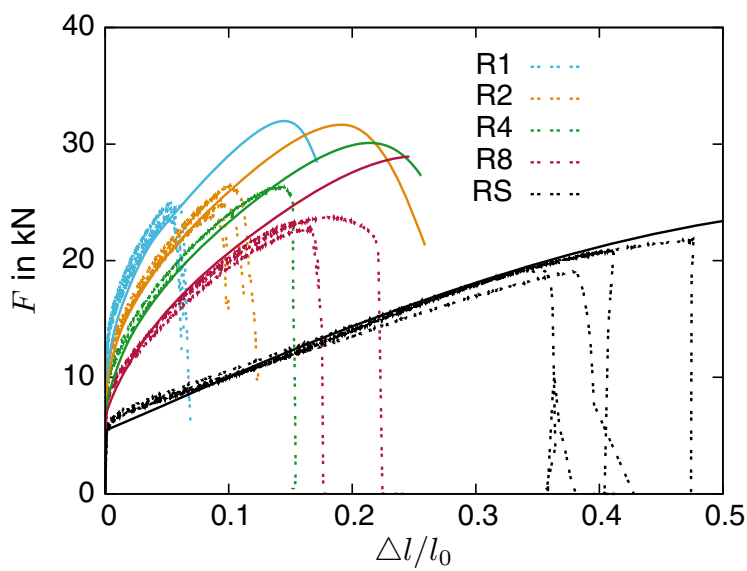

\subsubsection{Stress Analysis and Material Forces for Cracks in TRIP-steels}

In this section, the micromechanically motivated material model from Sect. 22.3.2 is applied for fracture mechanics investigations. The material parameters are based on experimental data of cast steel $\mathrm{X} 3 \mathrm{CrMnNi}$ 16-6-6, see Table 22.1 and references [20, 21, 53].

\subsubsection{Crack Tip Fields in Front of a Blunting Crack Tip}

In ductile materials, cracks tend to blunt during opening. Thereby, finite strains occur in front of the tip leading to characteristic shapes of the stress and strain fields. The phase transformation influences these crack tip fields and leads to some new characteristics, which are shown within this section.

From linear elastic fracture mechanics it is known, that the stresses become singular at the crack tip. The same holds for elastic-plastic fracture mechanics at small strains, compare the well established HRR-field [74, 75]. If plasticity and finite strains are considered, the situation is different. In this case crack tip blunting is incorporated, which means that an initially sharp crack deforms to a notch like shape. The stress fields are not singular any more. The stress component perpendicular to the surface of the blunted notch vanishes ( $\sigma_{11}$ in the ligament), because of the free surface. The crack opening stress $\left(\sigma_{22}\right)$ reaches a maximum value at a distance in front of the crack and a finite value at the surface of the notch, see McMeeking [76]. If the considered material has a very high work hardening capacity, it is possible that the distinctive $\sigma_{22}$ maximum does not occur. Instead the stress monotonically increases towards the crack tip and reaches high values there. This is reported by Yuan et al. [77] for an austenitic steel with pronounced strain hardening. 

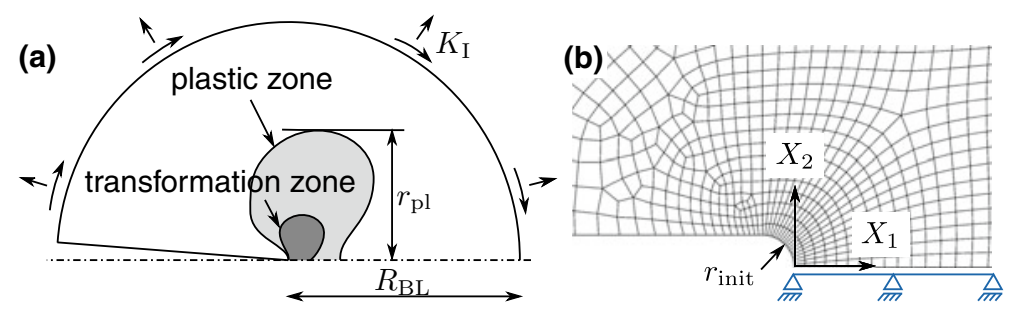

Fig. 22.6 a Sketch of the boundary-layer approach, b Detailed view of the initial crack tip and the finite element mesh together with the utilized coordinate system, reprinted from [20], with permission from Elsevier

In order to study the fields at a blunting crack without influence of a specific specimen geometry, small scale yielding under plain strain conditions is assumed. This is realized by a boundary-layer model, which is a circular region surrounding the crack tip, compare Fig. 22.6. Displacements known from the linear elastic $\mathrm{K}$-field are prescribed on the boundary. In order to ensure small scale yielding, zones of inelastic deformation have to be small compared to the radius $R_{\mathrm{BL}}$. In the case of strain induced phase transformation the transformation zone is always embedded inside the plastic zone, and thus $r_{\mathrm{pl}} \ll R_{\mathrm{BL}}$ is sufficient.

The boundary value problem is solved numerically employing finite elements. The boundary-layer model is discretized by quadrilateral elements with quadratic shape functions and reduced integration (ABAQUS: CPE8R). A zoomed view of the mesh near the crack is given in Fig. 22.6. It is typical to use a small initial radius at the crack tip in blunting studies, which is also shown in the figure. If the radius blunts to several times of its initial size, the resulting stress fields will be the same as for an initially sharp crack. The advantage of this initial radius is that distorted elements will show up later compared to the sharp crack tip. Further information can be found in [20].

The simulations yield steady state solutions for the mechanical fields. Thus, for example the distributions of stresses at different load levels $K_{\mathrm{I}}$ are self similar. They coincide in a single curve, if the distance to the initial crack tip in the reference configuration $X_{1}$ (Fig. 22.6) is normalized by $J / \sigma_{0}$, which is proportional to the crack tip opening displacement. Hereby, $J$ is the $\mathrm{J}$-integral and $\sigma_{0}$ is the initial yield stress of the material. Under small scale yielding and plane strain $J$ is related to the prescribed K-factor and the elastic constants via

$$
J=\frac{K_{\mathrm{I}}^{2}\left(1-v^{2}\right)}{E} .
$$

In order to point out the effect of the transformation hardening and the transformation strains, different model materials are considered, see Table 22.5. Figure 22.7 shows the corresponding true stress-strain curves and the evolution of martensite volume fraction. The variants are realized by an appropriate choice of the material 
Table 22.5 Investigated model materials-Overview

\begin{tabular}{l|l}
\hline Variant & Description, influence \\
\hline TRIP & TRIP-steel \\
$\Uparrow$ & Volumetric transformation strain \\
$\triangle_{\mathrm{V}}=0$ & $\begin{array}{l}\text { TRIP-steel without volumetric transformation } \\
\text { strain }\end{array}$ \\
$\Uparrow$ & Deviatoric transformation strain \\
$\boldsymbol{\epsilon}_{\text {trip }}=\mathbf{0}$ & $\begin{array}{l}\text { TRIP-steel without any transformation strain, } \\
\text { Transformation induced hardening }\end{array}$ \\
$\Uparrow$ & Austenite (without phase transformation) \\
Aust. &
\end{tabular}

(a)

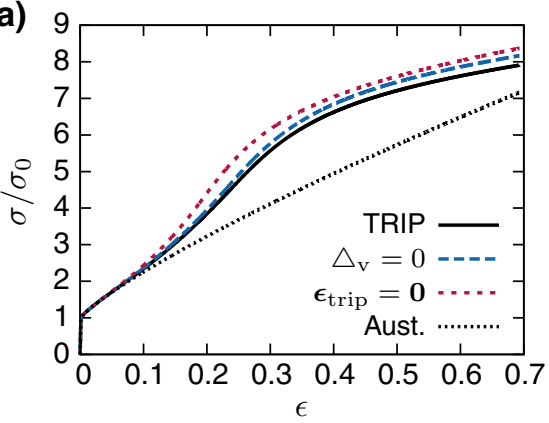

(b)

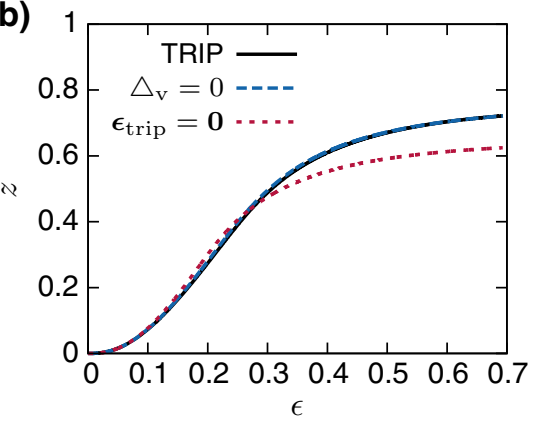

Fig. 22.7 Uniaxial behavior of the different model materials, a True stress-strain curves ( $\sigma$ vs. $\epsilon$ ), b Evolution of martensite volume fraction $z$ versus true strain $\epsilon$

parameters, compare [20]. On the one hand, there is the TRIP-steel showing the full phase transformation with corresponding hardening and straining. On the other hand, there is a non-transforming austenite as reference. In between, there are two variants of TRIP-steel to study the role of deviatoric and volumetric transformation strain: one without volumetric transformation strain $\left(\triangle_{v}=0\right)$, and the other one without any transformation strain $\left(\boldsymbol{\epsilon}_{\text {trip }}=\mathbf{0}\right)$.

At first, results for the TRIP-steel are discussed. The course of the stress components in the ligament is depicted in Fig. 22.8. Towards the crack tip (from right to left) an increase of the stress components is visible. The component $\sigma_{11}$ reaches a maximum at $X_{1}=0.23 \mathrm{~J} / \sigma_{0}$ and goes down to zero with smaller distance $X_{1}$. The stress component $\sigma_{22}$ increases continuously towards the tip and does not show a maximum at some distance in front of the tip. This is consistent with [77] for material exhibiting high strain hardening. Furthermore, the crack opening stress $\sigma_{22}$ shows a characteristic curvature, which is highlighted by the inflection points in Fig. 22.8. These characteristics are triggered by phase transformation. They occur in or near to the region with considerable martensite volume fraction, see Fig. 22.9. The stress triaxiality $h$, which is also depicted in Fig. 22.9, has a maximum in front of the tip and goes down to a finite non-zero value towards the crack. 


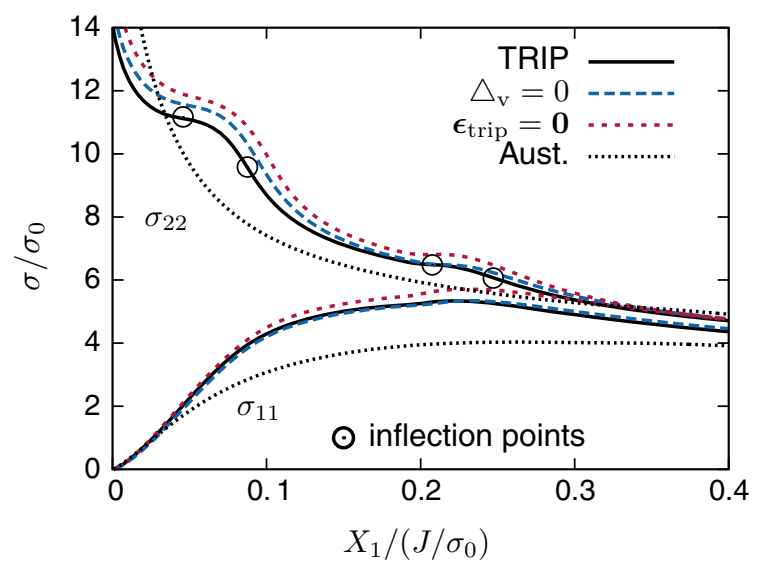

Fig. 22.8 Distribution of the stress components $\sigma_{11}$ and $\sigma_{22}$ in front of the blunting crack in the ligament for the different model materials, $X_{1} /\left(J / \sigma_{0}\right)$ is the normalized distance to the crack tip in the undeformed configuration

(a)

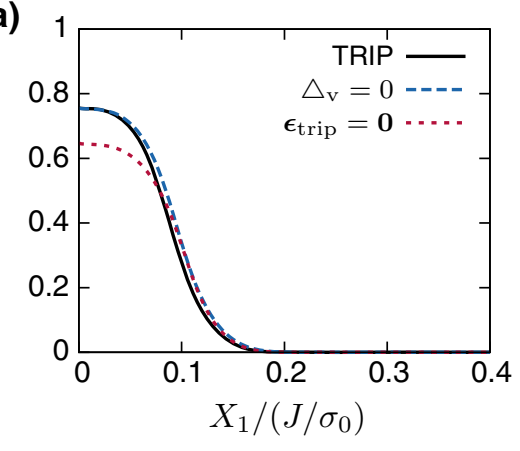

(b)

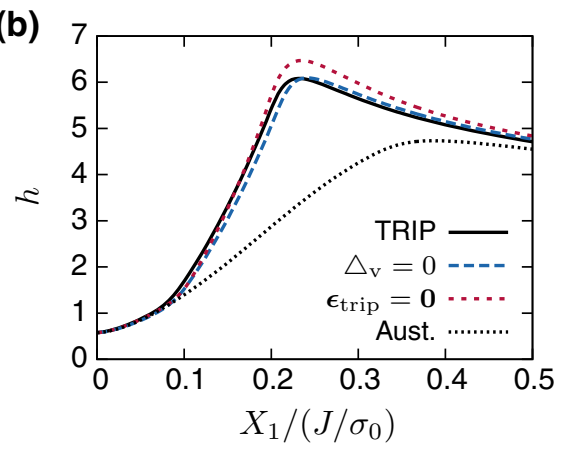

Fig. 22.9 Distribution of the martensite volume fraction $z$ (a) and stress triaxiality $h$ (b) in the ligament for the model materials, $X_{1} /\left(J / \sigma_{0}\right)$ is the normalized distance to the crack tip

In the next step the different model materials are taken into account. Comparing austenite to TRIP-steel, it becomes clear that phase transformation leads to higher stresses and stress triaxiality in the region near to the transformation zone. This is consistent with findings of Stringfellow [78]: By comparing TRIP-steel to nontransforming austenite, an increase of equivalent stress due to phase transformation was found. Considering the other two TRIP-variants one can conclude that the main effect comes from transformation induced hardening. The TRIP-steel without transformation strain already shows all the characteristic features (inflection points, increased stresses). The transformation strains reduce the stress components because of strain softening and affect the stress fields considerably near the tip, where the highest martensite volume fraction is present. 
Interestingly, there is a region very near to the crack tip, where the stresses are highest in the austenite. This is explained with the different hardening behavior of the model materials. It can already be seen in Fig. 22.7, that the TRIP-steel variants reach higher stresses, but the austenite has the higher hardening modulus at strains larger than 0.3. Asymptotically, the stress-strain curve of pure austenite leads to the highest stresses, which is observed in the crack tip fields.

The implications of these observations on fracture in TRIP-steels were discussed in detail by Burgold et al. [20]. In the case of cleavage, phase transformation could have a negative effect, because of the higher principal stresses. But there is an open question, how martensite affects the critical fracture strength. In the case of ductile fracture, the transformation has a positive impact on toughness, because the transformation induced hardening hinders the growth of micro voids. This is supported by the work of Hütter et al. [79], who studied the effect of hardening on the ductile fracture mechanism by micromechanical simulations. In both cases (ductile fracture and cleavage) a shielding effect due to phase transformation can occur, because martensite evolution dissipates mechanical work, which is not available for crack growth anymore.

\subsubsection{Material Forces in Consideration of Phase Transformation}

In this section the influence of phase transformation on the crack driving force in TRIP-steels and a possible shielding effect are investigated. Therefore, the theory of material forces (also called configurational forces) is applied, which are generalized thermodynamic forces acting on defects, see e.g. [80-82]. A great advantage of the approach is that it distinguishes between the material force acting on the crack tip and those occuring in zones of inelastic deformation, see e.g. [83-86]. Generally, a material force $\vec{G}_{\mathrm{D}}$ acting on defects in a domain surrounded by the contour $\Gamma$ reads

$$
\vec{G}_{\mathrm{D}}=\int_{\Gamma} \boldsymbol{Q} \cdot \vec{n} \mathrm{~d} S
$$

with the unit normal vector $\vec{n}$ and the energy-momentum tensor of elasto-statics $Q$ according to Eshelby, see [81]. The tensor $\boldsymbol{Q}$ is calculated by

$$
\boldsymbol{Q}=\psi \boldsymbol{\delta}-(\operatorname{grad} \vec{u})^{\mathrm{T}} \cdot \boldsymbol{\sigma}
$$

with the unit tensor of second order $\delta$.

In this section we assume small strains, isothermal and static conditions, and the absence of body forces. The small strain tensor can be additively decomposed into elastic and inelastic strains.

$$
\varepsilon=\varepsilon_{\mathrm{el}}+\boldsymbol{\varepsilon}_{\mathrm{trip}}+\boldsymbol{\varepsilon}_{\mathrm{vpl}}
$$



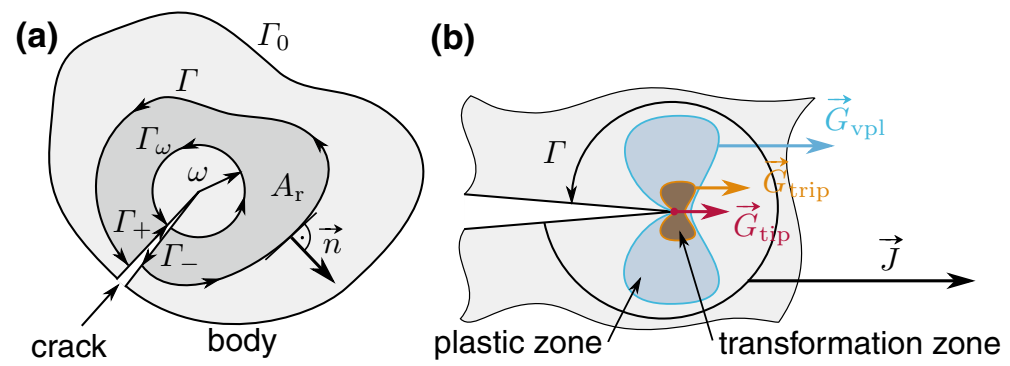

Fig. 22.10 a Cracked body with different contours and regular subpart $A_{\mathrm{r}}$, b Different material forces near a crack tip in TRIP-steel acting on different defects

The application of material forces to TRIP-steel has been discussed in detail by Kuna et al. [21]. Thereafter, a local balance of material forces is established by taking the divergence of $\boldsymbol{Q}$, which after some rearrangements leads to

$$
\operatorname{div} \boldsymbol{Q}+\boldsymbol{\sigma}: \operatorname{grad} \boldsymbol{\varepsilon}_{\mathrm{vpl}}+\boldsymbol{\sigma}: \operatorname{grad} \boldsymbol{\varepsilon}_{\text {trip }}+\left|\Delta g_{\text {chem }}^{\mathrm{a} \rightarrow \mathrm{m}}\right| \operatorname{grad} z=\overrightarrow{0} .
$$

The term $\Delta g_{\text {chem }}^{\mathrm{a} \rightarrow \mathrm{m}}$ describes the difference in the chemical energy of austenite and martensite and is known from Sect.22.2.2.4. Following (22.92) the divergence of $\boldsymbol{Q}$ is balanced by material body forces resulting from gradients of the internal variables $\boldsymbol{\varepsilon}_{\text {trip }}, \boldsymbol{\varepsilon}_{\mathrm{vpl}}$ and $z$. The gradient terms enter the derivation, because the gradient of the HELMHOLTZ potential $\psi$ is evaluated, see [21].

Furthermore, the global balance of material forces is achieved by integrating the local balance (22.92) over a regular subpart $A_{\mathrm{r}}$ of the body. Figure 22.10 depicts the case of a cracked body with its regular subpart (domain $A_{\mathrm{r}}$ ) and an enclosing contour $C=\Gamma+\Gamma_{+}+\Gamma_{-}-\Gamma_{\omega}$. Applying the divergence theorem to the integrated local balance and some rearrangements enable the formulation of the material force acting on the crack tip:

$$
\begin{aligned}
\vec{G}_{\text {tip }} & =\lim _{\omega \rightarrow 0} \int_{\Gamma_{\omega}} \boldsymbol{Q} \cdot \vec{n} \mathrm{~d} S=\vec{J}-\vec{G}_{\text {trip }}-\vec{G}_{\mathrm{vpl}} \\
\vec{J} & =\int_{\Gamma} \boldsymbol{Q} \cdot \vec{n} \mathrm{~d} S \\
\vec{G}_{\text {trip }} & =\lim _{\omega \rightarrow 0} \int_{A_{\mathrm{r}}}-\left(\sigma: \operatorname{grad} \boldsymbol{\varepsilon}_{\text {trip }}+\left|\Delta g_{\mathrm{chem}}^{\mathrm{a} \rightarrow \mathrm{m}}\right| \operatorname{grad} z\right) \mathrm{d} A \\
\vec{G}_{\mathrm{vpl}} & =\lim _{\omega \rightarrow 0} \int_{A_{\mathrm{r}}}-\sigma: \operatorname{grad} \boldsymbol{\varepsilon}_{\mathrm{vpl}} \mathrm{d} A
\end{aligned}
$$


The crack driving force $\vec{G}_{\text {tip }}$ is a J-integral evaluated over a vanishingly small contour $\Gamma_{\omega}$ surrounding the crack tip. It is computed by subtracting material forces $\vec{G}_{\text {trip }}$ and $\vec{G}_{\text {vpl }}$, which result from inelastic processes in the domain $A_{\mathrm{r}}$, from the usual J-integral vector $\vec{J} . \vec{G}_{\text {tip }}$ turns out to be a modified J-integral, which is path independent in the case of plastic deformations and phase transformations.

The different material forces relevant for the considered TRIP-steel are illustrated in Fig. 22.10. There is the usual J-integral describing the sum of material forces for all defects inside the contour $\Gamma$. The crack driving force $\vec{G}_{\text {tip }}$ acts directly on the tip. The material forces due to inelastic processes $\vec{G}_{\text {trip }}$ and $\vec{G}_{\text {vpl }}$ act on the transformation zone and the plastic zone, respectively. In the case, that $\Gamma$ only includes a part of these zones, the terms $\vec{G}_{\text {trip }}$ and $\vec{G}_{\mathrm{vpl}}$ are material forces acting on this part of the zones. As long as the inelastic material forces are positive, they decrease the available crack driving force, see (22.93), which indicates a shielding effect.

The material forces are computed numerically by finite elements within the software package ABAQUS. Therefore, postprocessing of the FE results is performed. With the help of the equivalent domain integral method, nodal material forces are formulated, compare [19, 21, 83]. The global material forces (22.93)-(22.96) are then achieved by a weighted summation of the nodal material forces.

In the following, material forces are evaluated for the example of small scale yielding under plain strain conditions. Again the boundary-layer model, Fig. 22.6, is used, this time without an initial radius at the tip. Material parameters and additional information concerning the numerical model can be found in [21].

A cohesive zone (ABAQUS built-in) is placed at the ligament in order to incorporate crack initiation and propagation. One reason is that the path independence of the crack driving force can be elaborated under non-proportional loading. The other reason is that the cohesive zone model $(\mathrm{CZM})$ contains a parameter $G_{0}$ called work of separation, which is exactly the dissipated work per area of crack extension. It is therefore the critical value of the crack driving force during crack extension, which has to be calculated correctly by the proposed material force method.

A bilinear traction separation relation is applied, which includes reversible opening of the cohesive zone until the cohesive strength $t_{0}$ is reached. The corresponding value of the separation is $s_{0}$. Loading beyond this point is connected to damage evolution and a descending load carrying capacity of the cohesive zone. At the separation $s_{\mathrm{t}}$ the cohesive zone is totally damaged and cannot carry tractions anymore. During the complete separation process until $s_{t}$, the work of separation $G_{0}$ is consumed.

The mesh consists of fully integrated quadrilateral elements with quadratic shape functions (ABAQUS: CPE8). The mesh design in the region of crack extension can be seen in the following figures.

A detailed view of different nodal material forces (notation: node $(K))$ is shown in Fig. 22.11. The upper crack face is depicted with the initial crack tip approximately in the center. A close scrutiny of these figures reveals, that there are substantial material forces at the nodes connected to the cohesive zone (nodes on the crack face right to the initial tip). Since the CZM incorporates the fracture process, these are nodal material forces acting on the smeared crack tip. Furthermore, there are 


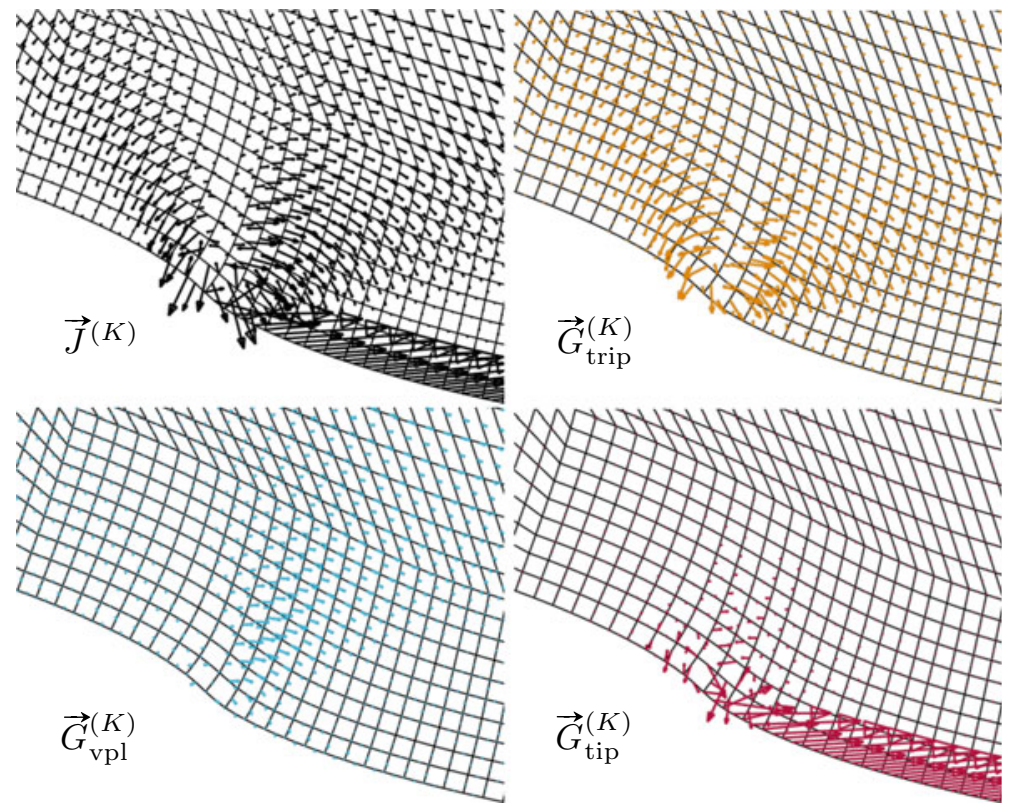

Fig. 22.11 Distribution of the different nodal material forces (node $(K)$ ), which are nodal contributions to the material forces of (22.93)-(22.96), the region near the initial crack tip (center of the crack flank) is shown during fracture initiation, cohesive elements are not shown here, reprinted from [21], with permission from Springer Nature

considerable nodal material forces in the bulk resulting from viscoplastic deformation and phase transformation. In contrast to the nodal contributions to the usual J-integral $\vec{J}^{(K)}$, the nodal contributions to the material force acting on the tip $\vec{G}_{\text {tip }}^{(K)}$ nearly vanish in the volume, because of the subtraction of the inelastic nodal material forces $\vec{G}_{\text {trip }}^{(K)}$ and $\vec{G}_{\text {vpl }}^{(K)}$. Small remaining vectors can be explained with numerical errors in the computation of the gradients of the internal variables. They have actually no influence on the material forces after summation of the nodal contributions, see the next paragraph.

The nodal material forces are summed up to get material forces acting on the crack, the plastic zone and the transformation zone. In the case of crack propagation under mode I only the $x_{1}$-components are non-zero. These components of the different material forces are plotted in Fig. 22.12. Firstly, plausibility is checked: The regular $\mathrm{J}$-integral $J_{1}$ equals the $\mathrm{J}$-integral $J_{1}^{\mathrm{SSY}}$, which is prescribed at the boundary layer, see again (22.88). Furthermore, once the cohesive zone is fully initiated, the crack driving force $G_{1}^{\text {tip }}$ equals the work of separation $G_{0}$ of the CZM. The symbols in the diagram mark start and end of the fracture initiation process. At $s^{\text {tip }}=s_{0}$, the first cohesive element reaches the cohesive strength and starts to damage. At $s^{\text {tip }}=s_{\mathrm{t}}$, the first cohesive element reaches total damage and for the first time $G_{1}^{\text {tip }}=G_{0}$ is 
Fig. 22.12 Evolution of the nonzero component of the material forces for the boundary-layer model, $J_{1}^{\mathrm{SSY}}$ is the prescribed J-integral (external loading), $G_{0}$ is the work of separation of the CZM, start and end of fracture initiation is marked by symbols, reprinted from [21], with permission from Springer Nature

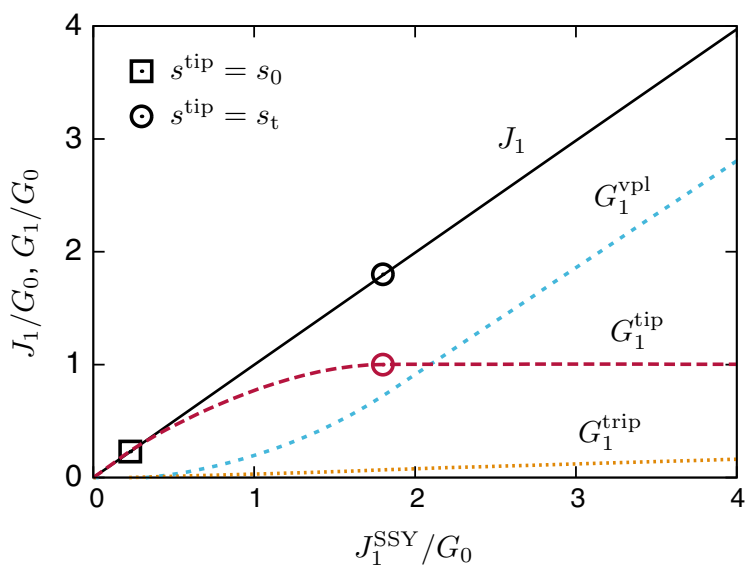

fulfilled. Thus, the proposed material force method is verified to give reasonable results.

Secondly, the contributions of the inelastic processes can be viewed in detail, Fig. 22.12. In contrast to the local distribution of the nodal material forces, the plasticity induced material force $G_{1}^{\mathrm{vpl}}$ is dominant compared to the transformation induced $G_{1}^{\text {trip }}$. This is because the plastic zone is much larger than the transformation zone in the example. Both contributions are positive, which underlines the shielding effect of viscoplasticity and martensitic phase transformation.

In Fig. 22.13, the path independence of the formulated crack driving force is demonstrated by evaluating $G_{1}^{\text {tip }}$ for different domains and comparing it to the usual J-integral $J_{1}$. Until the start of fracture initiation in the CZM, both values are equal. For the case of monotonically and proportionally loaded cracks, the classical J-integral is known to be approximately path independent even if inelastic material behavior is present. This changes during crack initiation: $J_{1}$ becomes path dependent whereas the material force acting directly on the crack tip $G_{1}^{\text {tip }}$ remains path independent.

\subsubsection{Damage and Fracture of High Alloy TRIP-steel}

Now, the simulation of damage and crack extension in high alloy TRIP-steel is elaborated in more detail. On the one hand, a cohesive zone model (CZM) is applied and appropriate cohesive parameters are estimated. On the other hand, damage evolution and crack growth is modeled by the phenomenological model, Sect. 22.3.3, including the developed non-local ductile damage formulation. 


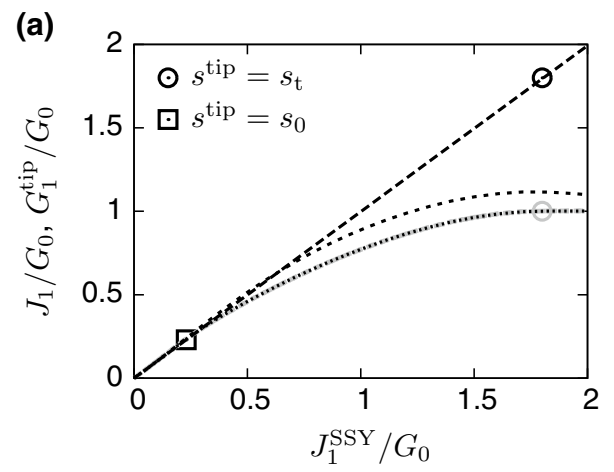

$$
\begin{array}{r}
G_{1}^{\text {tip }}: \text { domain } A_{1} \\
G_{1}^{\text {tip }}: \text { domain } A_{2} \\
G_{1}^{\text {tip }}: \text { global } \\
J_{1}: \text { domain } A_{1} \\
J_{1}: \text { doman...... } \\
J_{1}: \text { global } A_{2} \\
\cdots \cdots
\end{array}
$$

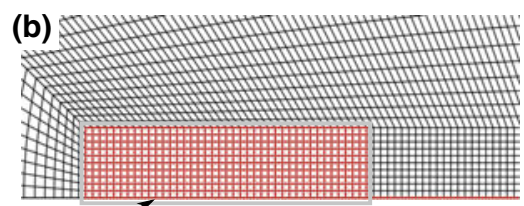

domain $A_{1}$ : one layer of nodes in the ligament

crack tip domain $A_{2}$ : $A_{1}+$ further marked nodes

Fig. 22.13 Demonstration of the path independence of the crack driving force $G_{1}^{\text {tip }}$, a Evaluation of the J-integral $J_{1}$ and $G_{1}^{\text {tip }}$ for different domains, $J_{1}^{\text {SSY }}$ is the prescribed J-integral, $G_{0}$ is the work of separation of the CZM, $\mathbf{b}$ Definition of the different domains used in the diagram, adapted from [21], with permission from Springer Nature

\subsubsection{Simulation of Crack Growth Using a Cohesive Zone Model}

In the last section, the versatility of the cohesive zone model for the simulation of crack extension was shown. In particular, the material parameter $G_{0}$ (work of separation) is the critical value of the material force acting on the crack tip. To apply this fracture criterion, it is important to identify $G_{0}$ for the given material.

In this section, the cohesive zone parameters are identified for the cast TRIP-steel $\mathrm{X} 2 \mathrm{CrNiMn} \mathrm{15-7-5}$. The chemical composition of the investigated steel is given in Table 22.1. The corresponding experiments are carried out on CT-specimens with chevron notch, see Fig. 22.14 and the paper by Burgold et al. [87] for further details. Fracture mechanical experiments on austenitic steels are a difficult task, because of the high work hardening capability and ductility. Excessive large scale yielding, which is shown in Fig. 22.14, impose challenges to the experiments and standard methods for measuring the crack length since compliance method or potential drop method do not work [87]. Therefore, special optical techniques (see Henkel et al. [88]) are used in order to measure crack extension $\Delta a$ and crack tip opening displacement CTOD $\delta$ at the surface of the specimen. From the experiments, force-displacement curves and crack growth resistance curves in terms of CTOD versus crack extension are obtained.

Because of large necking and thickness reduction during the tests three dimensional finite element calculations at finite strains are carried out, see [87, 89], 
(a)

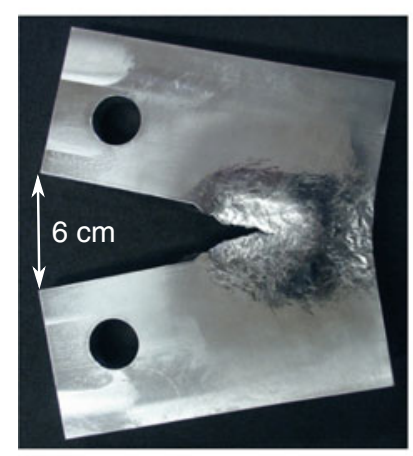

(b)

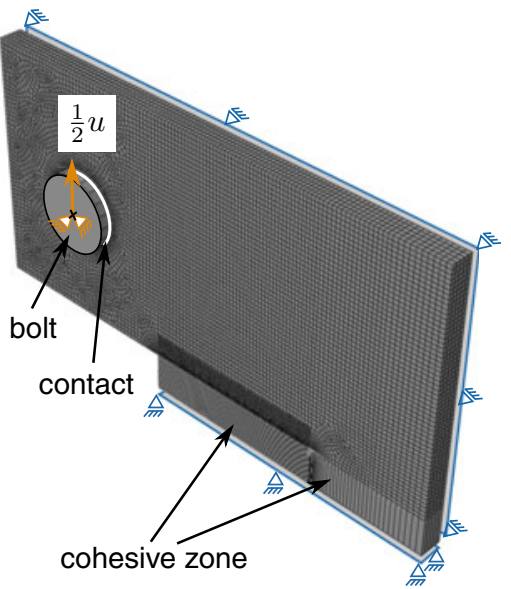

Fig. 22.14 a Deformed CT-specimen made of austenitic cast steel, b Finite element model with boundary conditions, displacement $u$ is prescribed on the bolt, reprinted from [87], with permission from Carl Hanser Verlag GmbH \& Co. KG, München

employing the commercial FE software ABAQUS. A quarter of the CT-specimen is modeled, compare Fig. 22.14. A vertical displacement $u$ is prescribed on the bolt, that is connected to the deformable body by a contact algorithm.

The utilized CZM was deveploped by Roth et al. [90, 91]. Hereby, a smooth exponential traction separation relation is applied, which has an adjustable shape due to two shape parameters $\hat{\varepsilon}$ and $\hat{\omega}$. One finding of the study is, that the choice $\hat{\varepsilon}=\hat{\omega}=1$ facilitates a good fit of the experimental data. Therewith, the well known traction separation relation from Xu and Needleman [92] is obtained.

The body is discretized by fully integrated continuum elements with linear shape functions (ABAQUS: C3D8). One layer of cohesive elements, which are user defined elements (UEL) [90, 91], is placed in the ligament. In contrast to ABAQUS built-in cohesive elements, the user elements work correctly on symmetry planes under large deformations.

It turned out to be sufficient to model the material behavior of the applied steel by a simplified constitutive law, in which transformation strains are not considered. The yield function of the rate independent model for isothermal conditions is given by

$$
y_{\mathrm{pl}}\left(\boldsymbol{\sigma}, \sigma_{\mathrm{y}}\right)=\sigma_{\mathrm{eq}}(\boldsymbol{\sigma})-\sigma_{\mathrm{y}} \leq 0
$$

with the VON MISES equivalent stress $\sigma_{\text {eq }}$ and the yield stress $\sigma_{\mathrm{y}}$. An associated flow rule is considered and the evolution equation for the plastic rate of deformation takes the form

$$
\boldsymbol{D}_{\mathrm{pl}}=\dot{\Lambda}_{\mathrm{pl}} \frac{\partial y_{\mathrm{pl}}}{\partial \boldsymbol{\sigma}}=\dot{\varepsilon}_{\mathrm{eq}} \boldsymbol{N}
$$


Fig. 22.15 Engineering stress-strain curves and evolution of martensite volume fraction $z$ under uniaxial tension, comparison between experimental data and simulation with the material model

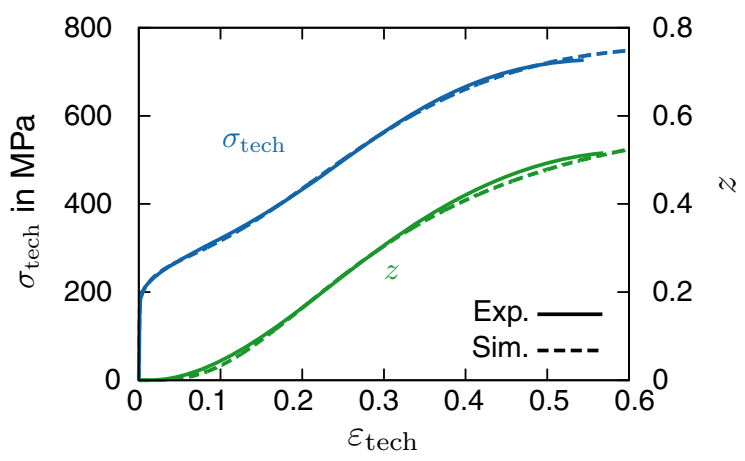

Hereby, the plastic multiplier $\dot{\Lambda}_{\mathrm{pl}}$ is equal to the rate of equivalent plastic strain $\dot{\varepsilon}_{\text {eq }}$. The tensor $\boldsymbol{N}$ is the flow normal known from (22.51).

The evolution of the yield stress $\sigma_{\mathrm{y}}$ is defined by the hardening law (22.71). This relation is simplified because the isothermal case is considered and LODE-angle dependence is not taken into account ( $r=\varepsilon_{\text {eq }}$ because $b=0$ in (22.73))

$$
\sigma_{\mathrm{y}}\left(\varepsilon_{\mathrm{eq}}, z\right)=\sigma_{0}+Z_{1} m_{\mathrm{z}}(z)+H\left(\varepsilon_{\mathrm{eq}}\right) \text {. }
$$

Thus, in addition to $Z_{1}$ the initial yield stress $\sigma_{0}$ becomes a material parameter. The work hardening term $H\left(\varepsilon_{\text {eq }}\right)$, see (22.85), is modified by $r_{\mathrm{c}} \rightarrow \infty$ and reads

$$
H\left(\varepsilon_{\mathrm{eq}}\right)=H_{0} \varepsilon_{\mathrm{eq}}^{q}
$$

with the parameters $H_{0}$ and $q$. The martensite influence term $m_{\mathrm{z}}(z)$ is given by (22.87) with the parameter $Z_{2}$.

The martensite kinetics is based on the empirical approach of Sect. 22.3.3.3. Therefore, the volume fraction of shear bands evolves as described by (22.48), in which $\alpha_{\mathrm{oc}}$ is directly taken as material parameter. The rate of martensite volume fraction is computed by (22.49) with $\beta_{\mathrm{oc}}$ and $n_{\mathrm{oc}}$ as additional material parameters.

The parameters of the material model are adequately calibrated to data from tensile tests of the cast TRIP-steel X2CrNiMn 15-7-5, see Fig. 22.15. In addition to the mechanical data, the martensite volume fraction $z$ is measured in situ by a novel magnetic device developed by Hauser et al. [93] and is applied in the calibration procedure. The identified parameters are given in Table 22.6.

Table 22.6 Parameters of the rate independent TRIP-steel model

\begin{tabular}{l|l|l|l|l|l|l|l}
\hline$\sigma_{0}$ & $H_{0}$ & $q$ & $Z_{1}$ & $Z_{2}$ & $\alpha_{\mathrm{oc}}$ & $\beta_{\mathrm{oc}}$ & $n_{\mathrm{oc}}$ \\
\hline $\mathrm{MPa}$ & $\mathrm{MPa}$ & - & $\mathrm{MPa}$ & - & - & - & - \\
\hline 153.8 & 434.2 & 0.401 & 370.9 & 2.06 & 5.66 & 0.988 & 3.805 \\
\hline
\end{tabular}



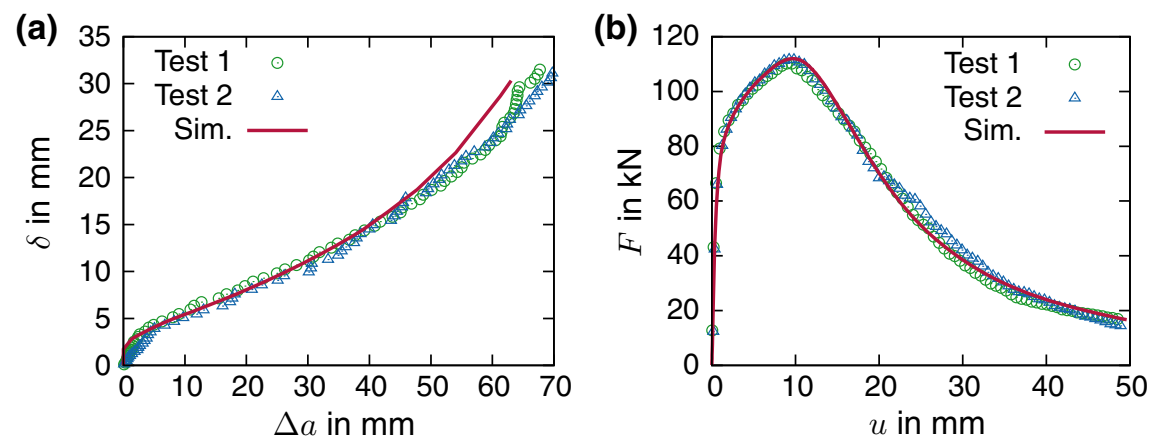

Fig. 22.16 Comparison of experiments and simulation, a Crack growth resistance curves in terms of CTOD $\delta$ and crack extension $\Delta a$, b Force-displacement curves ( $F$ vs. $u$ )

Table 22.7 Identified set of cohesive zone parameters

\begin{tabular}{l|l|l|l}
\hline$t_{0}$ & $G_{0}$ & $\hat{\varepsilon}$ & $\hat{\omega}$ \\
\hline $\mathrm{MPa}$ & $\mathrm{kJ} / \mathrm{m}^{2}$ & - & - \\
\hline 700 & 425 & 1 & 1 \\
\hline
\end{tabular}
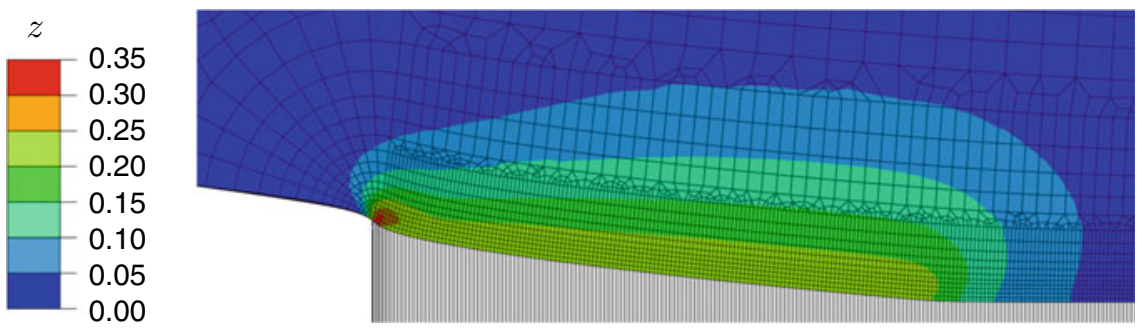

Fig. 22.17 Plot of the distribution of martensite volume fraction $z$ during crack extension, note the cohesive elements (white) in the ligament

Ultimately, the remaining independent cohesive parameters $G_{0}$ and $t_{0}$ have been chosen in a way, that the simulated force-displacement curve and crack growth resistance curve fit the experimental ones. The results are depicted in Fig. 22.16. Data from two realizations of the experiment are compared to the simulation with the final parameter set, which is given in Table 22.7. The two tests show only small differences and the simulation fits both tests very well. This supports the identified set of parameters and the whole modeling approach. Thus, the fracture process in the investigated CT-specimens is characterized by the given cohesive parameters. This concept was also successfully applied to a non-transforming austenitic steel in [87].

Finally, the martensite distribution around the crack is illustrated in Fig. 22.17. The highest amount (0.3-0.35) of martensite is formed during crack tip blunting near the 
initial crack tip. During crack extension a zone of martensite volume fraction between 0.2 and 0.25 is moved through the ligament.

\subsubsection{Simulations Using the Micromorphic Model of Ductile Damage}

Here, the micromorphic damage extensions of the phenomenological model from Sect. 22.3.3 will be used for simulation of ductile crack growth at isothermal conditions. Test results of the cast TRIP-steel X3CrMnNi 16-6-6 are utilized for calibration and validation. As described in [35], the calibration strategy for the considered class of damage models consists of three steps:

1. Choosing and fitting an isotropic strain hardening law to data of smooth and slightly notched tensile tests

2. Estimating an appropriate damage indicator function using FE-analysis and experimental results, e.g., of notched tensile tests

3. Fitting the damage parameters $\left(q_{1}-q_{3}, \varepsilon_{\mathrm{c}}\right.$ and $\left.L_{\mathrm{nl}}\right)$ to match results of a fracture mechanics test

For the first calibration step, the parameters of the strain hardening model for cast $\mathrm{X} 3 \mathrm{CrMnNi}$ 16-6-6 (Sect. 22.4.2) are slightly modified. This is done in order to improve the fit to the notched tensile tests discussed in Sect. 22.4.2.4.

For the second calibration step, undamaged FE-simulations of the notched tensile tests are performed to extract the loading history which leads to initiation of damage in critical regions, see [35]. As critical region, the center of the specimen at the symmetry plane is defined, since here the highest stress triaxiality occurs. The history until the rather sudden deviation of simulated and experimental force versus elongation curves is taken into account. The equivalent strain to damage initiation is plotted versus the stress triaxiality in Fig. 22.18. For all considered notch radii, a drop of triaxiality during deformation is observed, which is in contrast to recent results of a pressure vessel steel [35]. This behavior can be attributed to the strain hardening of the TRIPsteel: The high hardening capability delays a localization of deformation and the blunting of the notch decreases the triaxiality of the loading state. As indicator of damage initiation, the integral formulation

$$
\begin{aligned}
\omega\left(h, \varepsilon_{\mathrm{eq}}\right) & =\int_{0}^{\varepsilon_{\mathrm{eq}}(t)} \Omega(h) \mathrm{d} \varepsilon_{\mathrm{eq}}, \\
\Omega(h) & =\Omega_{1}+\Omega_{2} \exp \left(\Omega_{3} h\right)
\end{aligned}
$$

is used, which constitutes a modified Rice-Tracey-approach, see illustration in Fig. 22.18. The identified parameters read $\Omega_{1}=0.094, \Omega_{2}=0.666$, and $\Omega_{3}=$ 2.721 . 
Fig. 22.18 Damage initiation locus (equivalent plastic strain $\varepsilon_{\text {eq }}$ vs. stress triaxiality $h$ ): Loading history at the critical locations for the differently notched tensile tests, circles mark damage initiation; the damage initiation locus for constant triaxiality during loading is added as solid blue line

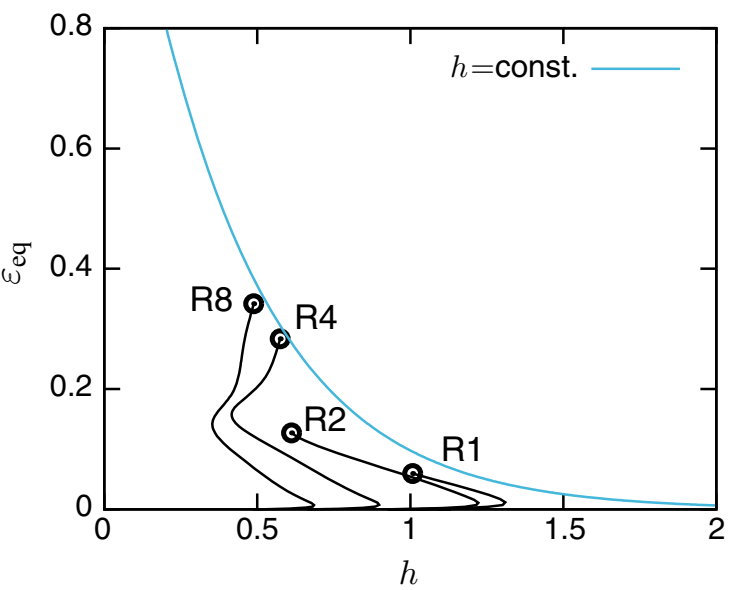

In addition to the notched tensile tests, fracture mechanics experiments are necessary in order to perform the third step of the proposed parameter calibration. Due to missing experimental results on fracture parameters of cast $\mathrm{X} 3 \mathrm{CrMnNi}$ 16-6-6, a numerical prediction serves as fracture mechanics reference. Appropriate parameters of a cohesive zone model are available for a similar steel in Sect.22.4.4.1. These results are utilized to make a realistic guess for the fracture behavior of $\mathrm{X} 3 \mathrm{CrMnNi}$ 16-6-6. A simulation of the 3D-CT-specimen is performed using the calibrated cohesive zone model from Sect.22.4.4.1 and the calibrated material model for $\mathrm{X} 3 \mathrm{CrMnNi}$ 16-6-6. Because fracture is completely described by the cohesive zone, damage of the bulk material is neglected. The obtained reference force versus displacement curve and the crack growth resistance curve in terms of CTOD versus crack length are shown in Figs. 22.19 and 22.21. The crack extension $\Delta a$ is evaluated using the maximum stress criterion with respect to the undeformed configuration at the surface of the specimen as explained in [94]. This criterion can be similarly evaluated for the cohesive zone model and the ductile damage approach.

The CT-specimen, see Fig. 22.14 and reference [87], is implemented as FE-model in ABAQUS. To avoid highly distorted elements, a small radius $r_{\mathrm{t}}=0.05 \mathrm{~mm}$ is applied at the crack tip as suggested in literature (see Fig. 22.20, [35, 76, 94]), which is permissible for the expected blunting prior to crack propagation. A 3D-finite element formulation employing reduced integration with quadratic shape functions for the displacement and linear shape functions for the micromorphic DOF are used (ABAQUS: C3D20RT). Along the ligament, a mesh size of $b_{\mathrm{e}} / L_{\mathrm{nl}}=0.25$ is prescribed, where $b_{\mathrm{e}}$ is the edge length of the element, see Fig.22.20. This recommendation can be found in literature to obtain converged results [34, 35, 95]. The axi-symmetric models of notched tensile tests are also meshed with the mentioned restrictions in regions of interest (ABAQUS elements: CAX8RT).

During the calibration process, the parameters $q_{1}$ and $q_{3}$ are fixed to the value 3 . Only the internal length $L_{\mathrm{nl}}$, the influence of equivalent strain $q_{2}$ (void nucle- 
(a)

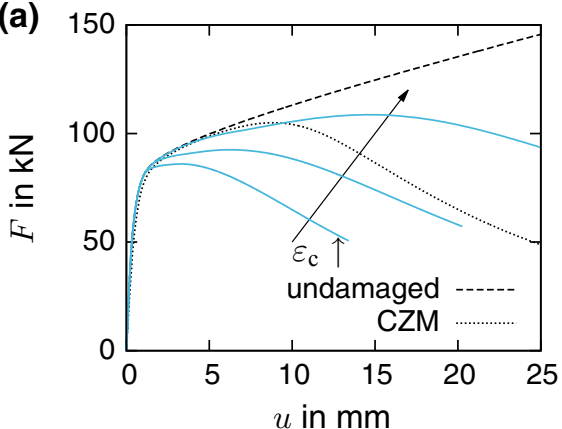

(c)

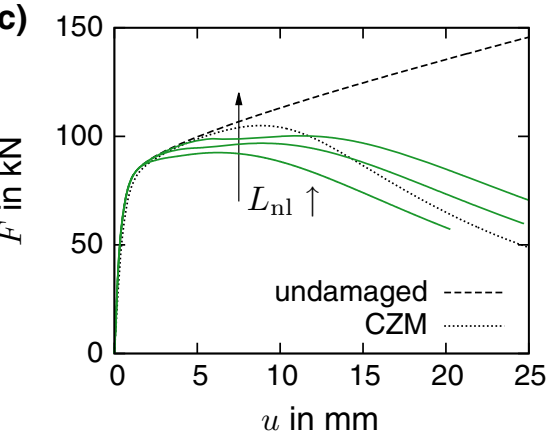

(e)

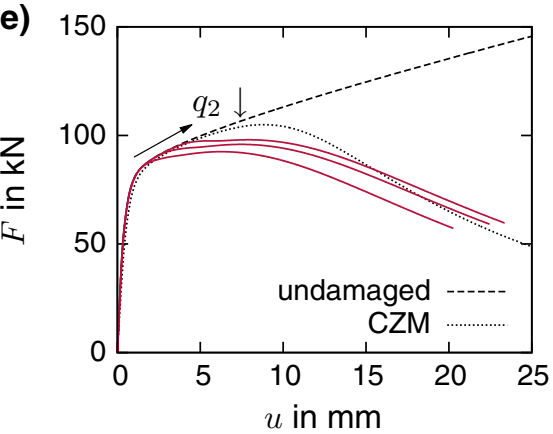

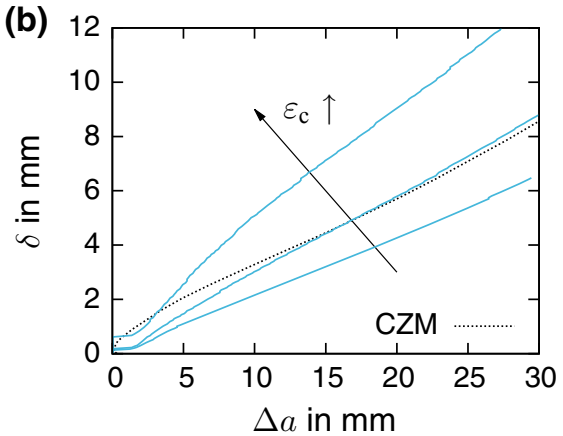
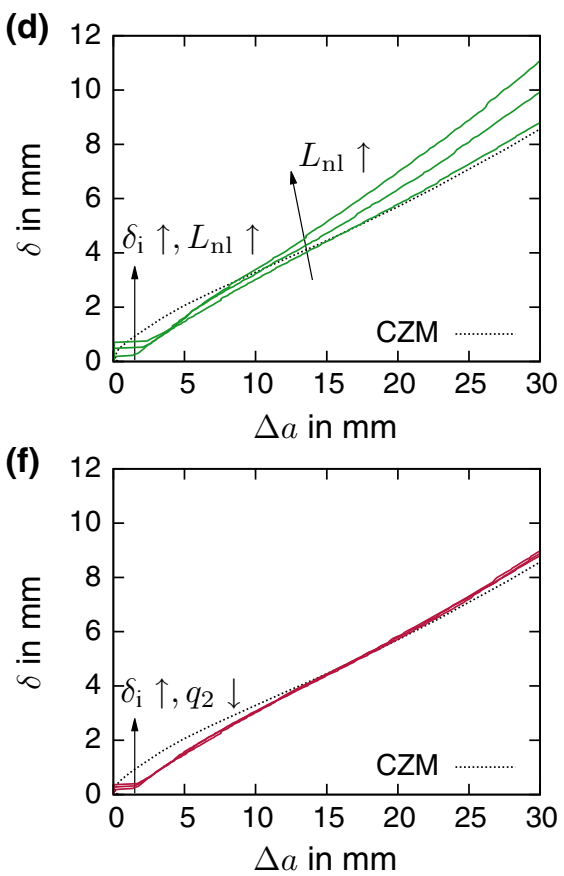

Fig. 22.19 Influence of different parameters of the damage evolution law on structural response of the CT-specimen (force $F$ vs. displacement $u$ curves, crack growth resistance CTOD $\delta$ vs. $\Delta a)$, Top row $(\mathbf{a}, \mathbf{b}): \varepsilon_{\mathrm{c}}=\{0.2,0.3,0.5\}$, Middle row $(\mathbf{c}, \mathbf{d}): L_{\mathrm{nl}}=\{0.5,0.75,1\} \mathrm{mm}$, Bottom row $(\mathbf{e}, \mathbf{f}): q_{2}=\{0.01,0.025,0.1\}$

ation), and the acceleration parameter $\varepsilon_{\mathrm{c}}$ are varied. Considerable small values are prescribed for $q_{2}$, because void growth is assumed as the main damage mechanism in the considered domain of moderate to high stress triaxialities.

In order to assess the influence of the free parameters, a sensitivity study is performed. The reference set of parameters is prescribed as: $\varepsilon_{\mathrm{c}}=0.3, L_{\mathrm{nl}}=0.5 \mathrm{~mm}$, and $q_{2}=0.1$. The results are summarized in Fig. 22.19 and compared to the reference cohesive zone simulation as well as the pure blunting solution: With varying $\varepsilon_{\mathfrak{c}}$, 
(a)

(b) $D$
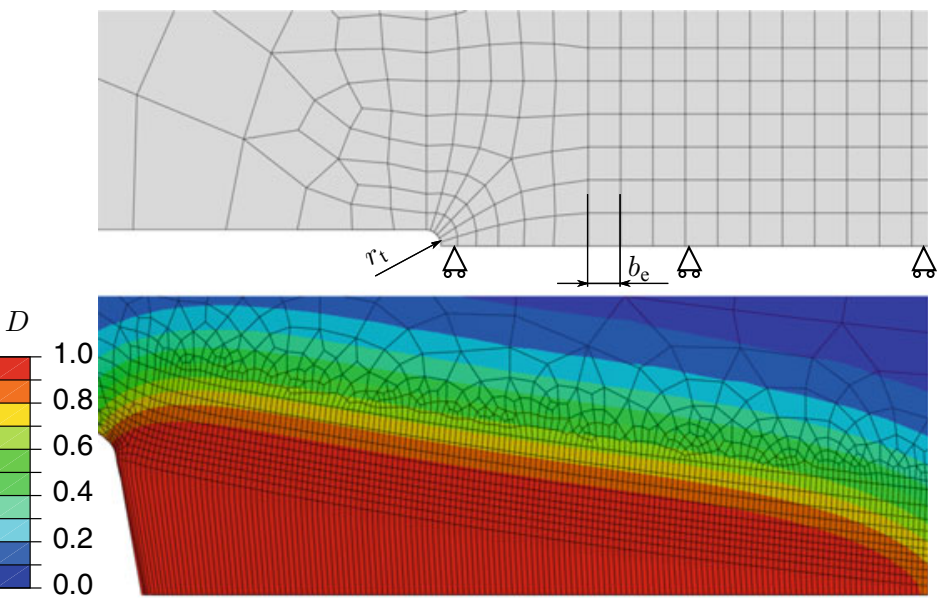

(c)

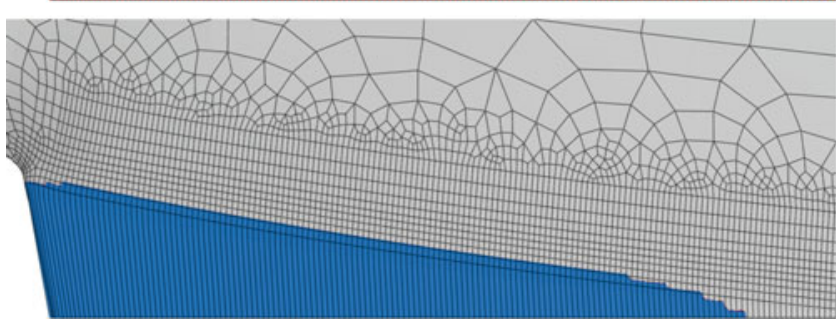

Fig. 22.20 Simulation of the CT-specimen, a Initial FE-mesh with crack tip rounding, b Deformed structure with damage distribution $D$ at a crack extension of $\approx 15 \mathrm{~mm}$, $\mathbf{c}$ Highlighted elements undergoing total damage

the slope of the crack growth resistance curve and the decreasing branch can be controlled. Simultaneously, the force level is changed. The internal length $L_{\mathrm{nl}}$ can be used to calibrate the maximum force and the crack initiation value, here the critical crack tip opening $\delta_{\mathrm{i}}$. Changing $q_{2}$ determines the deviation of the force response from the undamaged solution.

Motivated by the sensitivity study, a rough accordance is obtained with the manual calibration of the damage parameters: $\varepsilon_{\mathrm{c}}=0.2, L_{\mathrm{nl}}=1.0 \mathrm{~mm}$, and $q_{2}=0.01$. The response of the calibrated model is illustrated in Fig. 22.21. The location and amount of the maximum force need further improvement and the crack tip opening is underestimated. But the softening branch of the force versus displacement curve and the slope of the crack growth resistance curve are in acceptable accordance. It should be mentioned that a considerably large crack propagation can be modeled in a robust manner $(\Delta a=30 \mathrm{~mm})$; the simulations are interrupted externally after reaching the prescribed crack length. The contour plots in Fig. 22.20 show that damage is distributed over some layers of elements indicating the regularized character of the proposed damage model. The crack is represented by a layer of total 

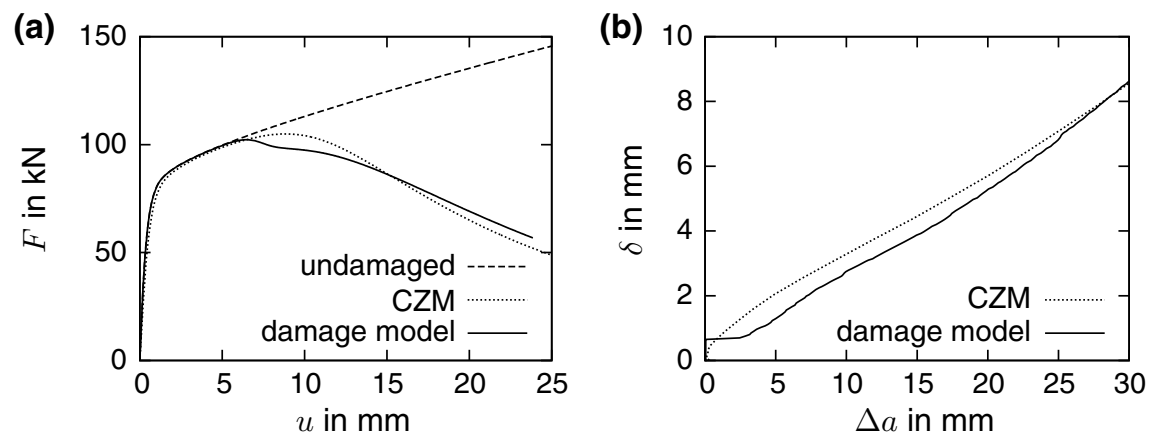

Fig. 22.21 Calibration results of micromorphic damage model with cohesive zone simulation (CZM) as reference, simulation without damage is added, a Force $F$ versus displacement $u$, b CTOD $\delta$ versus crack extension $\Delta a$

Fig. 22.22 Force $F$ versus elongation $\Delta l / l_{0}$ curves for notched tensile tests; solid lines correspond to the damage model prediction, dashed lines denote experiments

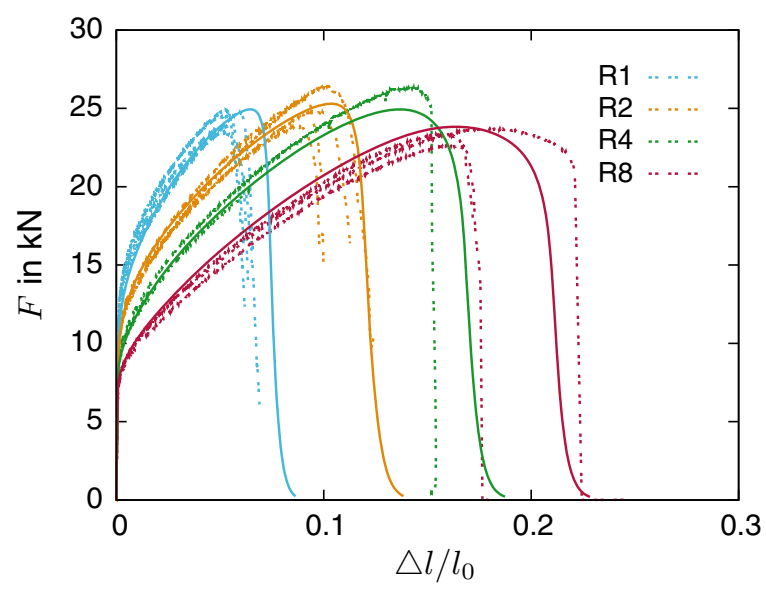

damaged, highly distorted elements. Hence, the applied patch solution for damping pathological widening of the damage zone works well in this special case.

With the estimated damage initiation and evolution parameters at hand, the notched tensile tests are simulated until failure. A comparison of predicted and experimental force versus elongation response reveals a reasonable agreement, see Fig. 22.22. The elongation at failure is slightly overestimated by the simulation for the small notch radii, but the load carrying capability is fully captured.

In conclusion, the proposed and calibrated micromorphic approach of ductile damage for high alloy TRIP-steel is able to reproduce the deformation, damage, and failure behavior including stable crack propagation. 


\subsection{Conclusions}

Based on a profound thermomechanical framework, a micromechanically based material model and a phenomenological approach are developed, which account for typical phenomena attributed to high alloy TRIP-steel: stress state dependent strain hardening, loading rate sensitivity and temperature dependency. In addition, a regularized ductile damage model, which is formulated with help of the micromorphic approach, is included.

For the phenomenological modeling approach, the applicability is demonstrated by calibration and validation with experiments of a cast $\mathrm{X} 3 \mathrm{CrMnNi} 16-6-6$ steel exhibiting strain induced $\alpha^{\prime}$-martensite formation. After calibrating the model to a subset of experiments (quasi-static loaded, uni-axial tension and compression tests at different temperatures), some predictive simulations are conducted. Firstly, predictions of stress-strain response at temperatures, which are not considered during calibration, are of reasonable quality. Especially, the disappearance of the tensionscompression asymmetry of strain hardening with increasing temperature is correctly reflected. Secondly, acceptable results are obtained for tensile tests at increased strain rates, where the thermomechanical coupling becomes important: The characteristic curve crossing of strain hardening curves is clearly captured by the model. Finally, simulations of notched tensile tests yield good agreement to available experiments. Hence, the model is also validated for inhomogeneous deformation.

With the help of the micromechanically motivated model, the effect of martensitic phase transformation on the loading situation at crack tips is investigated. The course of the stresses in front of a blunting crack shows characteristic curvature attributed to phase transformation. The most important influence is an increase in the stress components due to transformation induced hardening. Additional hardening affects ductile fracture in a positive manner, because the growth of microvoids is postponed. Another study examined the crack driving force for TRIP-steels under consideration of plastic deformation and phase transformation. By applying the theory of material forces a modified J-integral was formulated, which is path independent under these circumstances. Resulting from this, a shielding effect due to both inelastic strains was observed for small scale yielding.

Taking fracture process into account, a pragmatic cohesive zone model is successfully calibrated to reproduce crack propagation during loading of a CT-specimen. In combination with the cohesive zone, a simplified material model for isothermal and quasi-static conditions turned out to be sufficient to enable a good fit of the CT-tests. Finally, the micromorphic ductile damage model is applied to predict damage, crack propagation and failure of different structures made of a cast $\mathrm{X} 3 \mathrm{CrMnNi}$ 16-6-6 steel (CT-specimen, notched tensile tests). A reasonable agreement to experimental and numerical reference data is obtained after performing a developed calibration strategy.

Hence, versatile modeling tools for TRIP-steels are available, which are ready to be applied in simulations of future engineering applications. 
Acknowledgements The authors gratefully acknowledge the Deutsche Forschungsgemeinschaft (DFG, German Research Foundation) for funding the research in the framework of the Collaborative Research Center "TRIP-Matrix-Composite", (project number 54473466-SFB 799, subproject C5). In addition the authors thank the involved staff from SFB 799 and from the Institute of Mechanics and Fluid Dynamics (TU Freiberg) as well as Dr. Uwe Mühlich for their collaboration and numerous fruitful discussions.

\section{References}

1. H. Biermann, U. Martin, C.G. Aneziris, A. Kolbe, A. Müller, W. Schärfl, M. Hermann, Adv. Eng. Mater. 11, 1000 (2009)

2. D. Ehinger, L. Krüger, U. Martin, C. Weigelt, C.G. Aneziris, Steel Res. Int. 82, 1048 (2011)

3. D. Ehinger, L. Krüger, U. Martin, C. Weigelt, C.G. Aneziris, Int. J. Solids Struct. 66, 207 (2015)

4. C. Weigelt, C.G. Aneziris, H. Berek, D. Ehinger, U. Martin, Adv. Eng. Mater. 14(1-2), 53 (2012)

5. A. Jahn, A. Kovalev, A. Weiß, S. Wolf, L. Krüger, P.R. Scheller, Steel Res. Int. 82, 39 (2011)

6. S. Martin, S. Wolf, U. Martin, L. Krüger, Solid State Phenomen. 172-174, 172 (2011)

7. M. Linderov, C. Segel, A. Weidner, H. Biermann, A. Vinogradov, Mat. Sci. Eng. A 597, 183 (2014)

8. C. Ullrich, S. Martin, C. Schimpf, A. Stark, N. Schell, D. Rafaja, Adv. Eng. Mater. 21(5), 1801101 (2019)

9. S. Prüger, A. Seupel, M. Kuna, Int. J. Plast. 55, 182 (2014)

10. S. Wolf, S. Martin, L. Krüger, U. Martin, Mat. Sci. Eng. A 594, 72 (2014)

11. A. Seupel, R. Eckner, A. Burgold, M. Kuna, L. Krüger, Mat. Sci. Eng. A 662, 342 (2016)

12. A. Seupel, M. Kuna, in Proceedings of the 14th International Conference on Computational Plasticity - Fundamentals and Applications, COMPLAS 2017 (2017), pp. 576-587

13. M.P. Miller, D.L. McDowell, Int. J. Plast. 12(9), 875 (1996)

14. J. Serri, M. Martiny, G. Ferron, Int. J. Mech. Sci. 47(6), 884 (2005)

15. I. Papatriantafillou, M. Agoras, M. Aravas, G. Haidemenopoulos, Comput. Method. Appl. M. 195, 5094 (2006)

16. L. Mehlhorn, S. Prüger, S. Soltysiak, U. Mühlich, M. Kuna, Steel Res. Int. 82(9), 1022 (2011)

17. S. Prüger, L. Mehlhorn, S. Soltysiak, M. Kuna, Comp. Mater. Sci. 64, 273 (2012)

18. S. Prüger, L. Mehlhorn, U. Mühlich, M. Kuna, Adv. Eng. Mater. 15(7), 542 (2013)

19. A. Burgold, M. Kuna, S. Prüger, Procedia Mater. Sci. 3(0), 461 (2014). 20th European Conference on Fracture

20. A. Burgold, M. Kuna, S. Prüger, Eng. Fract. Mech. 138, 169 (2015)

21. M. Kuna, A. Burgold, S. Prüger, Int. J. Fract. 193, 171 (2015)

22. S. Prüger, Thermomechanische Modellierung der dehnungsinduzierten Phasenumwandlung und der asymmetrischen Verfestigung in einem TRIP-Stahlguss. Ph.D. thesis, TU Bergakademie Freiberg (2016). (in German)

23. S. Forest, J. Eng. Mech. 135(3), 117 (2009)

24. P. Germain, SIAM J. Appl. Mech. 25(3), 556 (1973)

25. B.D. Coleman, W. Noll, Arch. Ration. Mech. An. 13(1), 167 (1963)

26. J. Lemaitre, J.L. Chaboche, Mechanics of solid materials (Cambridge University Press, 1998)

27. H. Hallberg, P. Håkansson, M. Ristinmaa, Int. J. Plasticity 23, 1213 (2007)

28. J. Lemaitre, Nucl. Eng. Des. 80, 233 (1984)

29. H. Xiao, O.T. Bruhns, A. Meyers, Acta Mech. 124, 89 (1997)

30. H. Xiao, O.T. Bruhns, A. Meyers, Acta Mech. 138, 31 (1999)

31. A. Eshraghi, K.D. Papoulia, H. Jahed, J. Appl. Mech. 80(2), 0210271 (2013)

32. E. Diamantopoulou, W. Liu, C. Labergere, H. Badreddine, K. Saanouni, P. Hu, Int. J. Damage Mech. 26(2), 314 (2017) 
33. R.H.J. Peerlings, R. de Borst, W.A.M. Brekelmans, J.H.P. de Vree, Int. J. Numer. Meth. Eng. 39, 3391 (1996)

34. A. Seupel, G. Hütter, M. Kuna, Eng. Fract. Mech. 199, 41 (2018)

35. A. Seupel, M. Kuna, Int. J. Damage Mech. 28(8), 1261 (2019)

36. I. Tamura, Met. Sci. 16, 245 (1982)

37. G.B. Olson, M. Cohen, Metall. Trans. A 6A, 791 (1975)

38. R.G. Stringfellow, D.M. Parks, G.B. Olson, Acta Metall. Mater. 40(7), 1703 (1992)

39. Y. Tomita, T. Iwamoto, Int. J. Mech. Sci. 37(12), 1295 (1995)

40. T. Iwamoto, T. Tsuta, Y. Tomita, Int. J. Mech. Sci. 40, 173 (1998)

41. U. Ahrens, Beanspruchungsabhängiges Umwandlungsverhalten und Umwandlungsplastizität niedrig legierter Stähle mit unterschiedlich hohen Kohlenstoffgehalten. Ph.D. thesis, TU Paderborn (2003). (in German)

42. K. Nagayama, T. Terasaki, K. Tanaka, F.D. Fischer, T. Antretter, G. Cailletaud, F. Azzouz, Mat. Sci. Eng. A 308, 25 (2001)

43. F.D. Fischer, G. Reisner, E. Werner, K. Tanaka, G. Cailletaud, T. Antretter, Int. J. Plast. 16, 723 (2000)

44. M. Berveiller, F.D. Fischer (eds.), Mechanics of Solids with Phase Changes (Springer, 1997)

45. R. Mahnken, A. Schneidt, T. Antretter, Int. J. Plast. 25, 183 (2009)

46. R. Mahnken, A. Schneidt, Arch. Appl. Mech. 80, 229 (2010)

47. S. Prüger, M. Kuna, S. Wolf, L. Krüger, Steel Res. Int. 82(9), 1070 (2011)

48. R. Zaera, J.A. Rodríguez-Martínez, A. Casado, J. Fernández-Sáez, A. Rusinek, R. Pesci, Int. J. Plast. 29, 77 (2012)

49. H. Hallberg, P. Håkansson, M. Ristinmaa, Int. J. Solids Struct. 47(11-12), 1580 (2010)

50. A. Seupel, M. Kuna, Appl. Mech. Mater. 784, 484 (2015)

51. S. Martin, Deformationsmechanismen bei verschiedenen Verformungstemperaturen in austenitischem TRIP/TWIP-Stahl. Ph.D. thesis, TU Bergakademie Freiberg (2013). (in German)

52. S. Prüger, M. Kuna, Proc. Appl. Math. Mech. 11, 425 (2011)

53. S. Prüger, M. Kuna, K. Nagel, H. Biermann, Computational Plasticity XI Fundamentals and Applications 869-881 (2011)

54. P.P. Castañeda, P. Suquet, Adv. Appl. Mech. 34, 172 (1997)

55. F. Lani, Q. Furnémont, T.V. Rompaey, F. Delanney, P.J. Jaques, T. Pardoen, Acta Mater. 55, 3695-3705 (2007)

56. J.R. Rice, D.M. Tracey, J. Mech. Phys. Solids 17, 201 (1969)

57. A.L. Gurson, J. Eng. Mater. Technol. 44, 2 (1977)

58. M. Kuna, S. Wippler, Eng. Fract. Mech. 77, 3635 (2010)

59. N. Aravas, Int. J. Numer. Meth. Eng. 24, 1395 (1987)

60. A.C.F. Cocks, J. Mech. Phys. Solids 37(6), 693 (1989)

61. J. Besson, Int. J. Damage Mech. 19, 3 (2010)

62. D. Kulawinski, S. Ackermann, A. Seupel, T. Lippmann, S. Henkel, M. Kuna, A. Weidner, H. Biermann, Mat. Sci. Eng. A 642, 317 (2015)

63. S. Wolf, Temperatur- und dehnratenabhängiges Werkstoffverhalten einer hochlegierten CrMnNi-TRIP/TWIP-Stahlgusslegierung unter einsinniger Zug- und Druckbeanspruchung. Ph.D. thesis, TU Bergakademie Freiberg (2012). (in German)

64. A.M. Beese, D. Mohr, Acta Mater. 59, 2589 (2011)

65. C. Garion, B. Skoczen, Int. J. Damage Mech. 12, 313 (2003)

66. S.W. Yoo, C.S. Lee, W.S. Park, M.H. Kim, J.M. Lee, Comp. Mater. Sci. 50, 2014 (2011)

67. C.S. Lee, B.M. Yoo, M.H. Kim, J.M. Lee, Int. J. Damage Mech. 22(1), 95 (2012)

68. J. Lian, M. Sharaf, F. Archie, S. Münstermann, Int. J. Damage Mech. 22(2), 188 (2013)

69. J. Mediavilla, R.H.J. Peerlings, M.G.D. Geers, Comput. Method. Appl. M. 195, 4617 (2006)

70. B. Vandoren, A. Simone, Comput. Method. Appl. M. 332, 644 (2018)

71. L. Krüger, S. Wolf, U. Martin, P. Scheller, A. Jahn, A. Weiß, DYMAT 1069-1074 (2009)

72. L. Krüger, S. Wolf, S. Martin, U. Martin, A. Jahn, A. Weiß, P.R. Scheller, Steel Res. Int. 32(8), 1087 (2011)

73. R. Zaera, J.A. Rodríguez-Martínez, D. Rittel, Int. J. Plast. 40, 185 (2013) 
74. J. Hutchinson, J. Mech. Phys. Solids 16(1), 13 (1968)

75. J.R. Rice, G.F. Rosengren, J. Mech. Phys. Solids 16, 1 (1968)

76. R. McMeeking, J. Mech. Phys. Solids 25(5), 357 (1977)

77. H. Yuan, G. Lin, A. Cornec, Int. J. Fract. 71(3), 273 (1995)

78. R.G. Stringfellow, Mechanics of Strain-Induced Transformation Toughening in Metastable Austenitic Steels. Ph.D. thesis, Massachusetts Institute of Technology (1990)

79. G. Hütter, L. Zybell, U. Mühlich, M. Kuna, Comp. Mater. Sci. 80, 61 (2013)

80. M.E. Gurtin, Configurational Forces as Basic Concepts of Continuum Physics (Springer, New York, 2000)

81. R. Kienzler, G. Herrmann, Mechanics in Material Space (Springer, Berlin Heidelberg, 2000)

82. G.A. Maugin, Configurational forces: thermomechanics, physics, mathematics, and numerics (CRC Press, 2011)

83. T. Nguyen, S. Govindjee, P. Klein, H. Gao, J. Mech. Phys. Solids 53(1), 91 (2005)

84. B. Näser, M. Kaliske, R. Müller, Comput. Mech. 40(6), 1005 (2007)

85. N. Simha, F. Fischer, G. Shan, C. Chen, O. Kolednik, J. Mech. Phys. Solids 56(9), 2876 (2008)

86. O. Kolednik, R. Schöngrundner, F. Fischer, Int. J. Fract. 187(1), 77 (2014)

87. A. Burgold, S. Henkel, S. Roth, M. Kuna, H. Biermann, Mater. Test. 60(4), 341 (2018)

88. S. Henkel, D. Holländer, M. Wünsche, H. Theilig, P. Hübner, H. Biermann, S. Mehringer, Eng. Fract. Mech. 77(11), 2077 (2010)

89. A. Burgold, S. Roth, M. Kuna, Advances in Fracture and Damage Mechanics XVII 167-172 (2018)

90. S. Roth, G. Hütter, M. Kuna, Int. J. Fract. 188(1), 23 (2014)

91. S. Roth, Entwicklung und Implementierung zyklischer Kohäsivzonenmodelle zur Simulation von Werkstoffermüdung. Ph.D. thesis, TU Bergakademie Freiberg (2015). URL http://nbnresolving.de/urn:nbn:de:bsz:105-qucosa-209735. (in German)

92. X.P. Xu, A. Needleman, Modell. Simul. Mater. Sci. Eng. 1(2), 111 (1993)

93. M. Hauser, M. Wendler, S.G. Chowdhury, A. Weiß, J. Mola, Mater. Sci. Tech. 31(12), 1473 (2015)

94. G. Hütter, T. Linse., U. Mühlich, M. Kuna, Int. J. Solids Struct. 50, 662 (2013)

95. T. Linse, G. Hütter, M. Kuna, Eng. Fract. Mech. 95, 13 (2012)

Open Access This chapter is licensed under the terms of the Creative Commons Attribution 4.0 International License (http://creativecommons.org/licenses/by/4.0/), which permits use, sharing, adaptation, distribution and reproduction in any medium or format, as long as you give appropriate credit to the original author(s) and the source, provide a link to the Creative Commons license and indicate if changes were made.

The images or other third party material in this chapter are included in the chapter's Creative Commons license, unless indicated otherwise in a credit line to the material. If material is not included in the chapter's Creative Commons license and your intended use is not permitted by statutory regulation or exceeds the permitted use, you will need to obtain permission directly from the copyright holder.

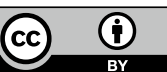

